\title{
The Non-Classical Nucleation of Crystals: Microscopic Mechanisms and Applications to Molecular Crystals, Ice and Calcium Carbonate
}

\author{
Richard P. Sear \\ Department of Physics, University of Surrey, Surrey GU2 7XH, United Kingdom
}

December 11, 2011

\begin{abstract}
Crystals form via nucleation followed by growth. Often nucleation data is interpreted using the classical theory of nucleation, which is essentially a simple theory for the nucleation of a fluid phase. I characterise this classical theory as making six assumptions; I discuss each assumption in turn. I then review experiments and simulations that find nucleation behaviour that cannot be described by the classical theory. The experiments are on the crystallisation from solution of molecules such as drugs and related molecules, ice and calcium carbonate. The review also covers work on non-classical nucleation in solutions of the protein lysozyme, and work on the fascinating phenomenon of nucleation induced by laser pulses. I hope this review will be of interest to those studying the crystallisation of both molecules and ions from solution. The review aims to advance our understanding of the crucial first step in crystallisation, and to enable researchers studying crystallisation in one system to learn from what others have done in studying analogous phenomena in different systems.
\end{abstract}

\section{Introduction}

This review is concerned with the initial stage of the formation of a crystal phase. This is the process called nucleation. It will not review crystal growth, although of course growth is also needed to obtain crystals and in practice in experiment it is often hard to separate nucleation from growth. Variations in nucleation rates can have important and beautiful effects, see Fig. 1. The review starts with the idea that there is a simple, widely used, theory for the nucleation of a new phase: classical nucleation theory. Then it reviews the literature on a number of molecular crystals, ice and calcium carbonate, where the nucleation behaviour appears to be more complex than the classical theory predicts. Here by molecular crystals we mean crystals of molecules such as aspirin, glycine, water etc, where van der Waals forces are important, although charges, hydrogen bonding etc may also be present. The review does not systematically cover ionic crystals, i.e., crystals where electrostatic interactions are dominant; it only covers in depth the crystallisation of calcium carbonate. Many other ionic crystals may have similarly complex behaviour but without more studies of nucleation in these systems, it is premature to review them. I will briefly mention protein solutions and colloidal suspensions but I will not systematically review them. An earlier review by the author covered these topics $^{1}$. This review will not consider metals or semiconductors. There is extensive coverage of both in Kelton and Greer's excellent book of $2010^{2}$. I hope that having read this review, the reader will have a better understanding of nucleation in across a wide range of systems. Although we have much still to learn about nucleation, a great deal of interesting and revealing work has been done, across a wide range of experimental systems. I believe there is a lot of scope for applying ideas and experimental techniques used in one complex crystallising system to other systems where the behaviour may be analogous. In particular, the formation of ice has been studied extensively over decades and so other fields can learn from what atmospheric scientists have discovered in studies of ice. 


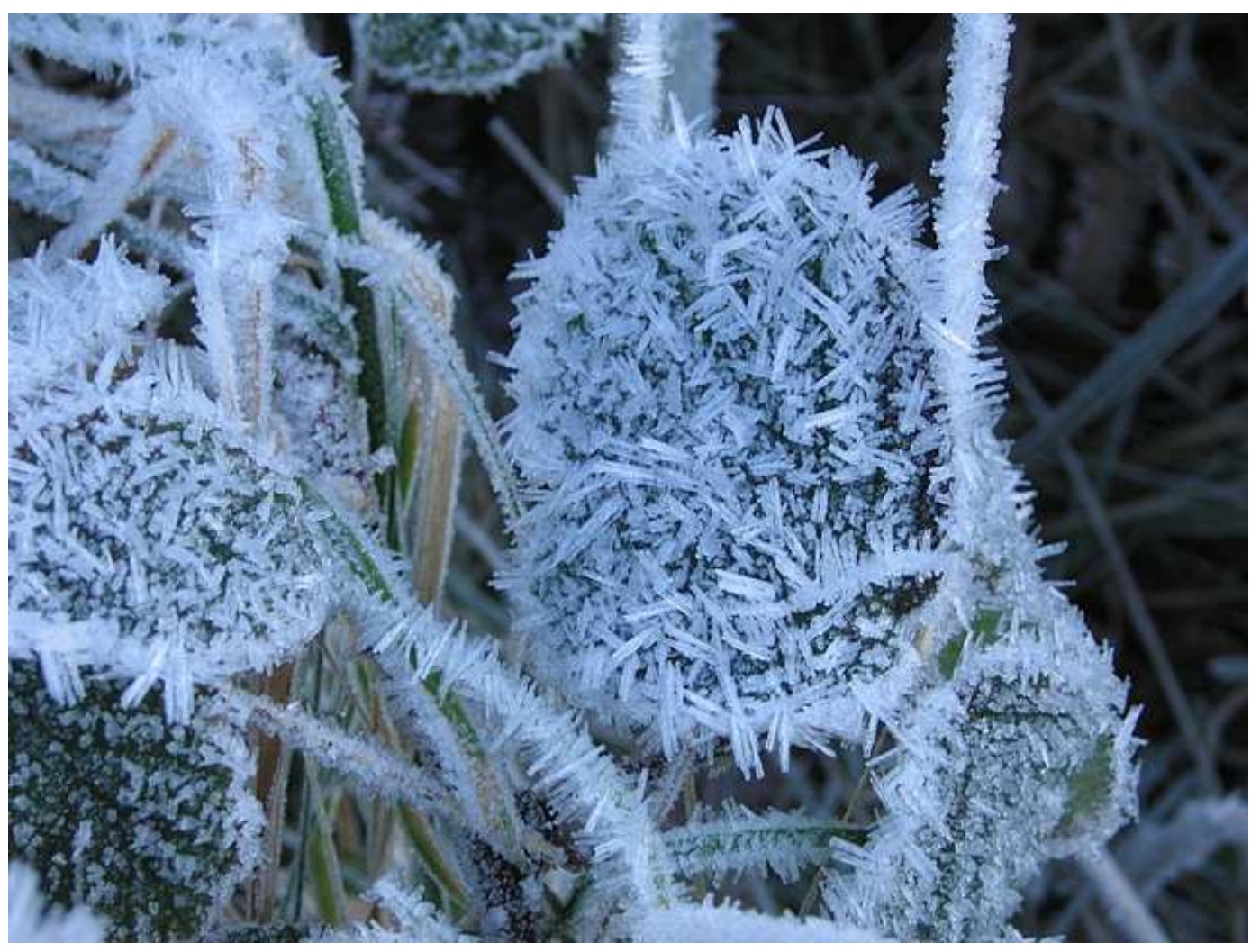

Figure 1: Ice crystals found by the Afon Ystwyth (Wales, United Kingdom). They are single crystals, meaning that each of them has a uniform crystal structure and orientation throughout, rather than consisting of many independently crystallised domains: A water molecule at one end of one of these about $8 \mathrm{~mm}$ long rods has the same orientation as one at the other end, with about 40 million identically aligned other water molecules in between. Such single crystal growth is rare as it occurs only if there is a shortage of crystal nuclei and it takes a long time - clean air and a cold spell of several days. The fact that the crystals are all about the same size suggests that nucleation occurred at the same time for all of them - the temperature must have dropped well below freezing rather quickly at the beginning of the cold spell. Copyright Rudi Winter and licensed for reuse under a Creative Commons Licence.

Below I will briefly review some of the assumptions of classical nucleation theory that may fail for some crystallising systems. See for example, Debenedetti's book ${ }^{3}$ for an introduction to this theory. Although this theory was developed for the nucleation of a new fluid phase, it is widely applied to crystallisation and may capture much of the physics in many systems. However, many molecular and ionic crystals nucleate in ways that are far from the simple classical picture. Indeed, as more systems are quantitatively studied we may find that a majority of systems cannot be described by the classical theory. This review will look at both experimental and computational work where there is evidence that nucleation is more complex than classical nucleation theory suggests.

Although there is a large literature on crystallisation there is still much to learn. There is also a gap between much of the simulation work, and experiment. We have learnt most of what we know about the microscopic dynamics of nucleation from simulation, but most of the simulation results we have are on homogeneous nucleation in models of very simple systems, e.g., noble gases. Experiments are done on more complex systems, such as drug molecules, and there nucleation is almost always heterogeneous. Also, our ability to understand our experimental results is greatly hindered by the fact that it is impossible to observe the nucleus in molecular and ionic systems. So here I will review experiments on heterogeneous nucleation, and simulation results mostly on homogeneous nucleation, but I hope that this review will encourage work to close the gap between 
experiment and simulation.

In this review I use heterogeneous nucleation to mean all nucleation that occurs with the nucleus interacting with any impurity, and all nucleation that occurs at any interface. This includes nucleation where the nucleus is in contact with a solid surface, and also where the nucleus includes, or has at its surface, impurity molecules or ions. It also includes nucleation far from any impurity but at a interface between two coexisting phases, e.g, nucleation of the crystal at a liquid/vapour interface. With this definition, nucleation is only homogeneous not heterogeneous when the nucleus is forming in the bulk far from any interface, and does not contain or interact with any impurity molecules or ions. See Fig. 2 for the effect of impurities of other amino acids on the crystallisation of glycine. Note that adding impurities can alter the polymorph that forms. However, it should be borne in mind that here, as is often the case with crystals, it is hard to separate the effects of impurities on nucleation from their effect on crystal growth. It seems likely that the impurities are affecting both processes here.

Nuclei are typically of between 10s to 1000s of molecules/atoms/ions, and so much of this review will be concerned with understanding the behaviour of aggregates of many more than one but much less than an infinite number of molecules or ions. So it is important to define carefully the words used to describe these finite aggregates. We will use the words cluster and occasionally nanoparticle to mean any compact aggregate of molecules or ions. In other words clusters and nanoparticles may be disordered or they may be crystalline. Also they may or may not be close to thermal equilibrium. If they are disordered they may be liquid like, with rapid internal dynamics of the molecules, or glassy, with arrested internal dynamics. If they are crystalline they may be close to equilibrium, or they may have defects or be in an metastable polymorphic state. As we will see, in some experiments we do not even know whether an aggregate is crystalline or not. I use the word nanoparticle interchangeably with cluster; a cluster of a few hundred or a few thousand molecules or ions will be nanometres across. In contrast to these more general terms, I use droplet to mean a cluster that is known to be in the liquid state, i.e., without crystalline ordering and with rapid internal dynamics (not glassy). I use crystallite to mean a cluster that is crystalline.

The structure of this review is as follows. I will start by enumerating the assumptions of classical nucleation theory that may fail for some crystallising systems. Then the majority of the review will consist of a number of relatively self-contained sections. Hopefully, each can be read more-or-less independently. Each section is devoted either to a substance with interesting and complex nucleation behaviour, or to a particular assumption of classical nucleation theory that appears to fail.

After the section that lists the assumptions underlying classical nucleation theory, the next 3 sections each cover an important example system. These are the drug ritonavir, ice and calcium carbonate. The sections after these 3 are each devoted to a class of non-classical nucleation behaviour. There are 6 sections devoted to nucleation behaviour that is complex due to an intermediate phase. This intermediate phase may be crystalline or fluid. It may be transient or it may form in large amounts.

Next the effects of defects in the crystal lattice are considered. These are not considered in the simple classical theory which is essentially a theory for the nucleation of a fluid phase - where there is no lattice and hence no defects in the lattice. There are then 2 sections on heterogeneous nucleation on solid surfaces. In experiments, nucleation seems to be almost always heterogeneous, and often occurs on the surface of an impurity particle. This can cause nucleation to deviate from the classical picture, e.g., due to an epitaxial match between the nucleating crystal and a crystalline surface. Finally, there is a section covering the interesting observation that nucleation can be induced by shining a laser on a solution, even though the wavelength of the laser is such that the solution does not significantly adsorb the light.

Crystallisation is a very large field, and inevitably I have had to be highly selective. I can only apologise to those authors whose work I was unable to cite. 


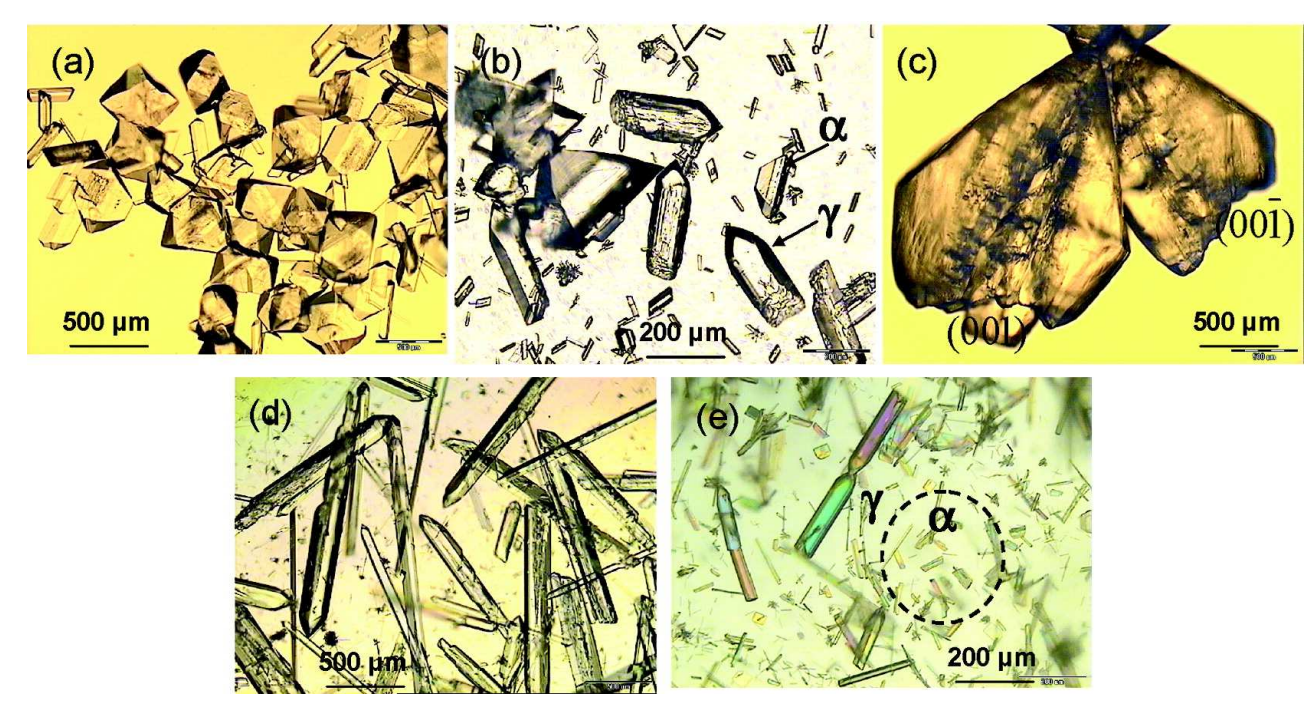

Figure 2: Glycine crystals obtained via crystallisation in the presence of small amounts of small molecule (comparable in size to glycine) impurities ${ }^{4}$. The crystals are obtained by Poornachary et al. ${ }^{4}$ by crystallisation from aqueous solution. The images are: (a) crystals of the $\alpha$ polymorph of glycine obtained with an impurity of $0.5 \mathrm{wt} \%$ (w/w glycine) of L-aspartic acid; (b) $\alpha$ and $\gamma$ polymorphs (2 wt \% of L-glutamic acid impurity); (c) $\gamma$ polymorph (4 wt \% of D-glutamic acid); (d) $\gamma$ polymorph (6 wt \% of L-aspartic acid); (e) $\alpha$ and $\gamma$ polymorphs (4 wt \% of L-aspartic acid). See Ref. 5 for glycine crystals without added impurities. Reprinted from Ref. 4 with permission. Copyright 2008 American Chemical Society.

\section{The Classical Theory for the Nucleation of Crystals}

I characterise the classical theory for the nucleation of crystals as making 6 assumptions:

1. Nucleation is a one-step process in which only one barrier is significant, and the nucleus consists of a microscopic piece of the new bulk crystal phase that is forming. This assumption has been known to be wrong in some systems for 100 years, and it caused Ostwald to propose his well-known rule of stages.

2. The nucleus grows one monomer at a time to the top and over the barrier.

3. The crystal lattice can be neglected and the nucleating phase essentially treated as if it were a droplet of fluid.

4. There is no other source of slow kinetics other than that due to the free energy barrier. Specifically, the microscopic kinetics are fast, there are no motions or rearrangements of the molecules that occur on a timescale comparable to the long timescale of nucleation.

5. The nucleation rate does not depend on the history of the sample, i.e., for a single-component sample it only depends on the temperature and pressure, not on, for example, the rate of cooling, or the thermal history of the sample.

6. Nucleation occurs over a saddle point in the free energy, i.e., the critical nucleus has a maximum free energy as a function of size but the free energy is a minimum with respect to other variables. For example, the critical nucleus of a fluid phase is expected to be close to spherical as this shape minimises the free energy at a given size.

If all these assumptions are valid then the nucleation rate is given, at least approximately, by classical nucleation theory. The homogeneous nucleation rate per unit volume is then ${ }^{1,3}$

$$
r=\rho j Z \exp \left(-F^{*} / k T\right)
$$


Here $\rho \exp \left(-F^{*} / k T\right)$ is the number of critical nuclei (= nuclei at the top of the barrier) per unit volume, $j$ is the flux of monomers onto a critical nucleus, and $Z$ is essentially the probability that the critical nucleus goes forward into the new phase not back into the metastable phase ${ }^{1} . F^{*}$ is the free energy change on forming a nucleus at the top of the barrier. Finally, $\rho$ is the number density of molecules of the nucleating species in the starting phase.

In this review, I have tried to cover all the possible breakdowns of the classical theory, that I know of. However, to keep the length of this review within a reasonable limit, I have chosen to deal in a partial way with the role of slow or glassy dynamics. This is assumption 4 , but such slow kinetics can also lead to assumptions 5 and 6 being violated. There is a large literature on glassy behaviour, and some of ideas there will need to be applied to the study of crystallisation in order to understand for example the competition between vitrification and crystallisation. A review covering both some of the literature on glassy dynamics and that on crystallisation in systems with these dynamics would be useful, but there is no space here for that.

Having summarised these assumptions, next there are three sections on substances where nucleation is more complex than the simple classical picture suggests.

\section{Ritonavir}

One of the most interesting recent examples of complex nucleation behaviour is that of ritonavir. Ritonavir is an anti-HIV drug. Like almost all substances, ritonavir has several polymorphs ${ }^{6,7}$. Ritonavir was "introduced in 1996. During development, only the polymorph Form I was found ${ }^{6}$. In 1998, a lower energy, more stable polymorph (form II) appeared, causing slowed dissolution of the marketed dosage form and compromising the oral bioavailability of the drug. This event forced the removal of the oral capsule formulation from the market." 7 . Ritonavir is a life-saving molecule so the disruption to its supply was highly undesirable. It was also of course very costly.

Polymorph control for drugs is crucial as different polymorphs dissolve at different rates and so enter the body at different rates (have different bioavailabilities) and so produce different doses. Therefore, regulatory approval to sell a drug is given for the drug in a specific polymorph, which must then be reliably produced. For an introduction to pharmaceutical crystallisation see the perspective of Variankaval et al. ${ }^{8}$.

Form II of crystalline ritonavir, although more stable, nucleates with difficulty, which is why it was at first missed. However, once it appeared, it was "contagious", "Within weeks this new polymorph began to appear throughout both the bulk drug and formulation areas ... solutions of ritonavir which although not saturated with respect to form I were $400 \%$ supersaturated with respect to form II ... this dramatically less soluble crystal form made this formulation unmanufacturable." Bauer $^{6}$ found that ritonavir in solution is predominantly in a conformation very different to that in form II. Nucleation may be slow due to some combination of slow kinetics for conformational change and a large interfacial tension between the polymorph form II and the solution. Whatever the reason, assumption 5 is clearly failing here,

The search for new drugs is leading to quite large molecules being considered. It has been suggested that this finding of a new polymorph relatively late in the drug-development process may become more common ${ }^{9}$.

This finding that a new, more stable, polymorph is "contagious" is strange at first sight but far from unique. Dunitz and Bernstein ${ }^{10}$ provide an entertaining list of much earlier examples of similar behaviour. For example, in the 1940s the molecule 1,2,3,5-tetra- $O$-acetyl- $\beta$-D-ribofuranose was studied ${ }^{10}$. It initially formed what was called polymorph $\mathrm{A}$. Then a second polymorph, polymorph $\mathrm{B}$, appeared, and in the presence of even traces of $\mathrm{B}$, polymorph $\mathrm{A}$ transformed rapidly to $\mathrm{B}$, and so labs contaminated with even undetectable traces of B could not be used to produce polymorph A. It was reported that a sample of polymorph A made in Cambridge (UK) was shipped to a lab in New York. The New York lab contained samples of B. The sample then rapidly transformed to the B form. 

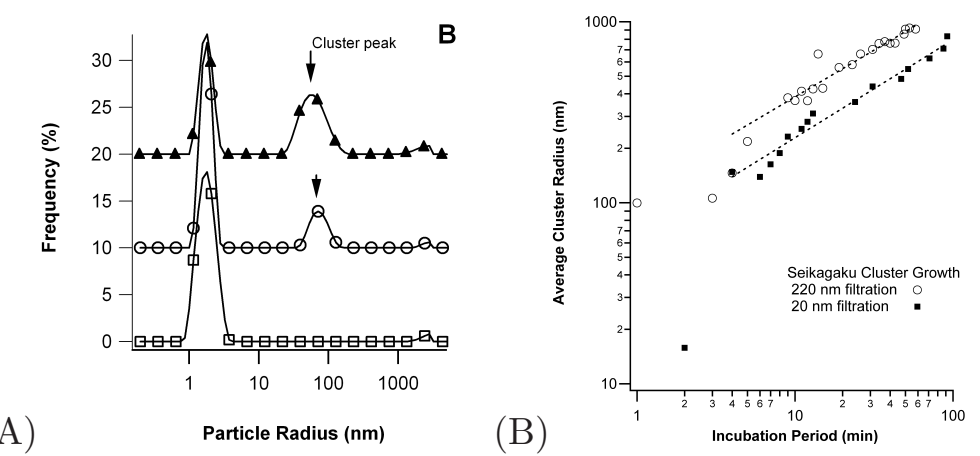

Figure 3: (A) Particle size distributions derived from dynamic light scattering ${ }^{11}$. The black triangles, open circles and open squares are all lysozyme but from 3 different manufacturers; from Sigma, Seikagaku and Worthington respectively. The peak at $2 \mathrm{~nm}$ is from monomers of the protein lysozyme. Note the peaks corresponding to lysozyme clusters centred near $90 \mathrm{~nm}$ in both the Seikagaku and Sigma lysozyme samples. This sub-micron peak is completely absent from this batch of Worthington lysozyme. For clarity, the size distributions for Seikagaku and Sigma lysozyme were offset from the origin. Note: peak amplitudes are proportional to the intensity of light scattered by the particle, not their number densities. (B) Cluster growth kinetics in supersaturated lysozyme solutions. Log-log plot of the evolution with time of the cluster radius for supersaturated Seikagaku solutions in the presence (open circles) or absence (black squares) of pre-existing clusters. The black-square sample was filtered using a $20 \mathrm{~nm}$ filter prior to the experiment. Cluster radii were taken from the particle size distributions obtained from dynamic light scattering. The dashed lines are fits through the data for $t \geq 5$ min. Adapted from Ref. 11 with permission. Copyright 2007 Elsevier.

The molecular events that underlie this remarkable behaviour are unknown. A possible explanation is the presence of seeds that promote the nucleation of a particular polymorph ${ }^{10}$. Here a seed is operationally defined as being a microscopic object that can survive under conditions where the bulk crystal melts/dissolves, and then when the solution is supercooled promotes the nucleation of a particular polymorph. The fact that such a seed can be formed the first time a polymorph is crystallised suggests that these seeds contain a microscopic crystallite of the appropriate polymorph, stabilised by an impurity of some sort. For example, a crystallite could be stabilised by being in a microscopic crack in a solid impurity, and so when the bulk crystal dissolves the crystallite in the crack remains to act as a seed. This would be a possible mechanism for violating assumption 5 .

As Dunitz and Bernstein ${ }^{10}$ already pointed out, the problem with proving or disproving this picture in experiment, is that such seeds can be tiny, perhaps as small as a few nanometres across. This means they are extremely difficult to detect, can easily enter the atmosphere in a lab, get caught in lab coat etc., and could potentially be produced in large numbers. However, in principle seeds could be studied in computer simulation to see if seeds that matched the above description could be made.

In experiments on the protein lysozyme in solution, see Fig. 3, Parmar et al. ${ }^{11}$ used light scattering to detect objects around $100 \mathrm{~nm}$ across. In this case they were irreversibly-formed aggregates of lysozyme itself. They then used filtration to remove them, and light scattering again to watch them grow back, see Fig 3. Removing the $100 \mathrm{~nm}$ objects dramatically affected the nucleation behaviour. This use of light scattering to detect small sub-micron seeds may be generally useful in studying crystallisation from solution. It is possible that in some systems, the time until crystals are first seen is at least partly due to the time for aggregates to grow large enough to support nucleation. This is different from the classical-nucleation-theory prediction, where the time is the waiting time until a rare fluctuation occurs. 


\section{Ice}

The phase transitions of $\mathrm{H}_{2} \mathrm{O}$ are the ones we all come across in our daily lives, and the formation of ice is the most important crystallisation process of all. See Fig. 1 for single crystals of ice that have formed by a river in Wales. We need to understand it to understand the Earth's climate. We are interested in both the freezing of supercooled water and the formation of ice crystals in wet air below $0^{\circ} \mathrm{C}$. Here I will focus on the formation of ice from cold wet air, as it appears it is often nonclassical. In particular it is often a two-step process - it occurs via the formation of water droplets. Below $0^{\circ} \mathrm{C}$, we are below the the triple point and so the liquid state is never the equilibrium phase, but particularly just below $0^{\circ} \mathrm{C}$, liquid water is only a little less stable than ice, so at all but low supersaturations with respect to ice, the cold wet air is supersaturated with respect to both ice and water.

For an introduction to the extensive literature on this topic see for example, the atmospheric physics textbooks of Seinfeld and Pandis ${ }^{12}$, Pruppacher and Klett ${ }^{13}$, and Mason ${ }^{14}$. The Pruppacher and Klett book has the most detailed presentation. More recent are the 2009 review of Hegg and Baker ${ }^{15}$, and the slightly older review of Cantrell and Heymsfield ${ }^{16}$. These works should be consulted for comprehensive reviews of ice formation. I do not attempt to comprehensively review experiments or simulations on the formation of ice, that would require a separate large review. See Fig. 4 for ice crystals growing inside water droplets.

Here I will do 3 things. The first is to summarise some well-known results on the nucleation of ice, much of this is from the atmospheric-science literature. Here, I hope to convince scientists who do not study the atmosphere, that there are useful ideas to be learnt from decades of study of ice formation. The crystallisation of water has been studied much more extensively than has the crystallisation of any other molecule. For example, it was known that crystallisation can occur via a metastable liquid phase many years before this was systematically studied in solutions of proteins and other molecules.

The second part of this section on ice considers the interesting phenomenon of contact nucleation. The third part is devoted to a brief assessment of the state of the art in the computer simulation of the nucleation of ice. After difficulties with inaccurate models, and with advances in computer power, simulation is now in a position to significantly advance our understanding of the freezing of water. I will discuss these 3 aspects of the nucleation of ice in order.

\section{The nucleation of ice from cold wet air}

Ice nucleates from wet air both under conditions where water can form droplets as the air is supersaturated with respect to water, and under conditions where the air is supersaturated with respect to ice but undersaturated with respect to water ${ }^{13}$. Both the nucleation of liquid water and of ice are always or almost always heterogeneous, they occur on particles ${ }^{12-14}$.

Indeed, despite many studies, see for example Cantrell and Heymsfield's review ${ }^{16}$, it is not clear that the homogeneous nucleation of ice in supercooled water has ever been observed in experiment. As water droplets have been studied down to temperatures below $-40^{\circ} \mathrm{C}^{16}$, it seems clear that rate of homogeneous nucleation of ice is low up to at least $-40^{\circ} \mathrm{C}$. It is possible than the homogeneous nucleation of water may in fact only occur at appreciable rates at much lower temperatures. See Murray et al. ${ }^{17,18}$ for some recent work on the nucleation of ice in water. Note that at low temperatures, it may be the cubic-ice, $\mathrm{I}_{c}$, polymorph of water that nucleates first and then transforms to the equilibrium polymorph of ice, hexagonal ice, $\mathrm{I}_{h}$. Also note that in water, extensive experimental work over decades has found that it is extremely difficult to eliminate heterogeneous nucleation, which is needed in order to study homogeneous nucleation. There is no obvious reason to expect that water is unique here, so presumably showing that nucleation is homogeneous in other molecular or ionic systems will be hard or impossible. Thus, experiments may be limited to studying heterogeneous nucleation.

In studies of ice nucleation in the Earth's atmosphere, the particles it occurs on are called iceforming nuclei (IN). Three classes of ice nucleation are recognised: "(1) contact nucleation occurs 
when an IN initiates freezing at the surface of a drop, at the highest temperatures; (2) immersion nucleation occurs when an IN is fully immersed and initiates freezing from within the volume of a drop, several degrees below the contact nucleation temperature; (3) deposition nucleation occurs when water vapour forms ice directly on the surface of a IN" ${ }^{19}$. Note that nucleation pathways (1) and (2) are for the nucleation of ice from metastable water droplets that have formed in the atmosphere at temperatures below $0^{\circ} \mathrm{C}$. It has been known for many years that ice can form via the condensation of liquid water droplets that then freeze ${ }^{13,14,20}$.

For nucleation class (3) no bulk water is seen but this does not mean that water plays no role in the nucleation of ice. Only a nanoscale amount of water present for a fraction of a second is required for the ice nucleus to form in water not air. This implies that water may be affecting ice nucleation even when it is not observed, and indeed when it is not stable in the bulk, i.e., when the air is undersaturated with respect to liquid water. Even if the wet air is undersaturated with respect to liquid water, nanoscale droplets of water can still form in cracks in the surface of a particle, via capillary condensation. These droplets can dramatically accelerate nucleation, see the section on nucleation in wedges and pits. This effect has been suspected for many years: "The appearance of some ice crystals slightly below water saturation ... because they [ice nuclei] may have acquired liquid water either by being hygrosopic 'mixed' nuclei or by capillary condensation" 14 .

Particles of different substances cause the nucleation of ice at different temperatures, some are more active than others in the sense that they induce freezing at higher temperatures ${ }^{13,16}$. However, we have almost no ability to predict if a particular substance will be good at inducing ice nucleation, or not. We do not understand the process sufficiently well to make predictions.
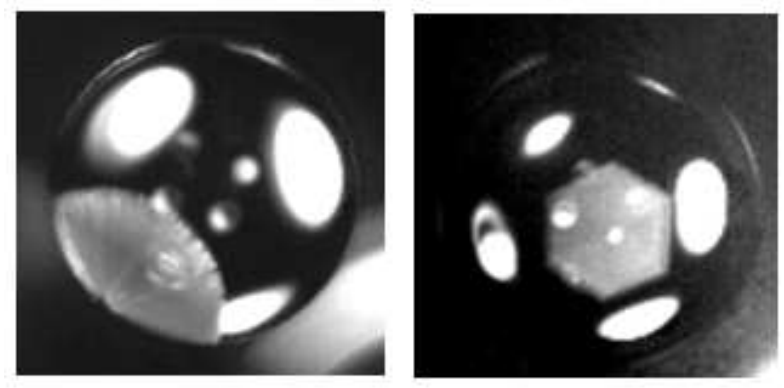

Figure 4: Two images of ice crystals growing in $10 \mu \mathrm{L}$ spherical-cap water droplets, viewed from above $^{21}$. The temperature $T \simeq-18^{\circ} \mathrm{C}$. The droplets are on the surface of an atomically-smooth silicon wafer. The images have been enhanced for contrast, with the freezing front appearing in grey and very bright reflections evident. In the left-hand image the crystal has nucleated on the surface of the silicon wafer at or near the contact line. In the right-hand image the crystal has nucleated at the silicon surface but away from the contact line. In the right-hand image the nucleated crystal is oriented with the basal plane aligned with the imaging plane, thereby showing a hexagonal shape, but this is not necessarily the preferred orientation. The images are courtesy of R. Shaw, see Ref. 21 for the movie of which these images are a frame.

\section{Contact nucleation}

Here we will consider number (1) of the three distinct mechanisms identified for atmospheric ice nucleation ${ }^{19}$. A number of experimental studies have found that under many conditions a water droplet freezes at a higher temperature if it is in contact with a particle than if the same particle is completely immersed in the water droplet ${ }^{13,14,22,23}$. If a droplet contacts a solid surface then the water/vapour interface will meet the particle surface along a line. At this line three phases meet, and so it is called a three-phase line. It has been found that nucleation can occur along this line ${ }^{13,14,22-25}$ in preference to in the bulk of the liquid or at either the water/vapour or water/solid interfaces. 
Suzuki et al. found that the temperature at which nucleation occurs at the contact line varies with contact angle, but that the dependence is not a simple monotonic function of contact angle ${ }^{24}$. They also found that nucleation occurs at higher temperatures on rougher, more heterogeneous surfaces $^{25}$. The roughness here was on the nanoscale. However, very recent work by Gurganus et $a l .{ }^{21}$ found that, in their system, nucleation did not occur preferentially along the contact line. It occurred in their water droplets mostly in contact with the substrate surface. See Fig.4 for images of crystals that have apparently nucleated near or at the contact line (left-hand image) and away from the contact line but in contact with the surface on which the droplet is resting (right-hand image).

It is not clear why nucleation seems to occur along the contact line in most experiments but not in those of Gurganus et al. ${ }^{21}$. Clearly, further work is required to answer this question, and to understand nucleation both at a surface and at a contact line. I have just two comments.

The first is that classical nucleation theory suggests that we should find what Gurganus et al. ${ }^{21}$ found: Nucleation at a solid surface but away from the contact line. As Tabazadeh, Djikaev and Reiss ${ }^{26,27}$ have emphasised in the context of homogeneous nucleation, the free energy of an ice nucleus will be lower at the water/vapour interface of a drop than in the bulk, unless all the surface planes of the ice crystallite surface melt. If even one surface does not, it will be favourable for ice to nucleate with that side at the water/vapour interface. Now, water apparently surface melts on all crystal facets, and not only near the melting temperatures but also at significantly lower temperatures ${ }^{28-30}$. Therefore the ice nucleus is expected to avoid the water/vapour interface and hence the contact line, which is what Gurganus et al. ${ }^{21}$ find. Note that this conclusion relies on neglecting line tension ${ }^{31}$ effects. The surface melting of ice implies that bulk ice is separated from vapour by a layer of liquid water, and pushing an ice nucleus from bulk water to the interface with the vapour phase would increase the free energy of the nucleus.

The second comment is that nucleation may be occurring at the contact line due to effects not considered in simple estimates of the free-energy barrier to nucleation. For example, if the droplet is being cooled, then the temperature will not be completely uniform in the system. Is the temperature then lowest along the contact line? Also, impurities are known to tend to concentrate at interfaces. The same physics that concentrates them there could also pull them to contact lines where they could speed nucleation. A set of experiments in which a parameter (cooling rate? impurity level? surface roughness?) is varied and the system goes from nucleation at the contact line to nucleation away from this line, would be very helpful.

\section{Computer simulation of ice nucleation}

Most of our information on the microscopic details of how nucleation occurs has come from computer simulation. These simulations are mostly of simple model potentials, such as the Lennard-Jones potential. Unfortunately, the computer simulation of ice nucleation is very difficult. Efforts have been hindered by the problem of obtaining a simple yet accurate model for water. Accurate here means predicts a reasonable value of the freezing temperature at atmospheric pressure, and correctly predicts that water will freeze into ice $\mathrm{I}_{h}$ (hexagonal ice), which is the ice polymorph that is the equilibrium phase of real water below $0^{\circ} \mathrm{C}$ at atmospheric pressure. The scale of the problem may be judged from the fact that the widely used TIP3P model of water has ice $\mathrm{I}_{h} /$ water coexistence at $-133^{\circ} \mathrm{C}$ at a pressure of 1 atmosphere, and at this pressure the ice II polymorph is more stable ${ }^{32,33}$ ! It should be noted that ice has many polymorphs that appear at higher pressure. The problem of developing a simple model that accurately predicts the phase diagram is discussed by Vega et al. ${ }^{32}$, and by Molinero and Moore ${ }^{34}$.

Not all models for water are as poor at predicting the liquid/crystal phase behaviour as TIP3P. The TIP4P model for water is better ${ }^{32,33}$. For this model, ice $\mathrm{I}_{h}$ is the stable polymorph at atmospheric pressure and water/ice coexistence is at a relatively good $-41^{\circ} \mathrm{C}$. Clearly this model cannot be used to predict experimental results, which are all at around $-40^{\circ} \mathrm{C}$ and above, but simulations could still provide insight into the microscopic mechanism of nucleation in ice. However, even with better models there are still challenges associated with simulations, for example, with the order 
parameters used in simulation to distinguish the growing crystal from the surrounding liquid ${ }^{35}$.

Perhaps the best computer simulation study of the nucleation of ice is that of Quigley and Rodger ${ }^{36}$. They studied the homogeneous nucleation of ice in the TIP4P model at $-97^{\circ} \mathrm{C}$, at atmospheric pressure. They found that cubic ice, ice $\mathrm{I}_{c}$, nucleated not ice $\mathrm{I}_{h}$, and that the barrier to nucleation of ice $\mathrm{I}_{c}$ was $79 k T$. It may be that the ice $\mathrm{I}_{c} /$ water interfacial tension is less than the ice $\mathrm{I}_{h} /$ water interfacial tension, promoting cubic ice nucleation.

Now, although the phase diagram of the TIP4P model is reasonable $32,37,38$, it is clear that the model is not accurate enough that we can be certain that the prediction of ice $\mathrm{I}_{c}$ nucleating in preference to $\mathrm{I}_{h}$ is correct. However, ice nucleation cannot be observed in experiment so we also cannot rule out the nucleation of ice $\mathrm{I}_{h}$ occurring via $\mathrm{I}_{c}$. For example, consider the following scenario: $\mathrm{I}_{c}$ nucleates in supercooled water, then $\mathrm{I}_{h}$ nucleates on a surface of the $\mathrm{I}_{c}$ crystallite when it is microscopic, and then $\mathrm{I}_{h}$ then grows much faster than $\mathrm{I}_{c}$. If this occurs then only $\mathrm{I}_{h}$ would be observed even though it was $\mathrm{I}_{c}$ that actually nucleated in the liquid.

Current experiments cannot rule out this possible mechanism and it is difficult to see advances in experimental techniques being sufficient to observe the nucleus in the near future. It is presumably around $10 \mathrm{~nm}$ across and crosses the barrier in much less than $1 \mathrm{~s}$. However, better models are being developed ${ }^{32,34}$. When these are combined with modern simulation techniques, we should be able to obtain improved simulation results for the homogeneous nucleation of ice. Moore and Molinero ${ }^{39}$ have already studied crystallisation in a model (called $\mathrm{mW}$ ) fitted to the phase diagram of water, including the freezing temperature at atmospheric pressure. They studied crystallisation at very large supercoolings via direct simulation and found that their system froze into a mixture of cubic and hexagonal ice.

This is all for the homogeneous nucleation of water, which is rarely if ever how water actually freezes. The study of heterogeneous nucleation at a surface requires a potential for the water/surface interaction. Such simulations will be required in order to understand experiments, but it may be some time before quantitative simulations of the heterogeneous nucleation of ice are performed.

\section{Calcium carbonate}

In this section, we will consider the crystallisation of calcium carbonate. Some other ionic systems may crystallise via mechanisms that are similar, However, ionic crystals are diverse and the crystallisation of many may follow the classical path. For example, the nucleation of the crystalline phase of sodium chloride from the melt has been studied by simulation by Valeriani et al. ${ }^{40,41}$. They found that the nucleus is quite close to the classical-nucleation-theory prediction. It was simply a microscopic piece of the bulk crystal. However, for example, the crystallisation of calcium phosphate may have aspects that are similar to the much more extensively studied calcium carbonate system ${ }^{42}$.

A recent book, Mesocrystals and Non-classical Crystallization by Cölfen and Antonietti ${ }^{43}$, and an extensive review by Meldrum and Cölfen ${ }^{44}$, have both reviewed "non-classical crystallisation" in calcium carbonate solutions in great detail. These works discuss in detail the unconventional or nonclassical crystals that are sometimes produced from solutions of calcium carbonate and other systems. Much of the work is motivated by a desire to understand how living organisms use confinement, controlled solution conditions, and specific proteins to control the crystallisation of calcium carbonate with amazing precision. This is part of the field called biomineralisation, see Mann's book ${ }^{45}$ for an introduction to biomineralisation. Thus, many, but not all, of these non-classical crystals are produced in the presence of one or more other species.

In general we cannot disentangle the roles of nucleation and growth in producing the crystals discussed by Antonietti, Cölfen and Meldrum. Hence the term "non-classical crystallisation" not non-classical nucleation. Thus, although the underlying nucleation behaviour may be interesting, we will not discuss it here. I will only discuss some recent experimental work that hints that nucleation may not classical, and also recent simulation work.

Simulating charged species in aqueous solution is very challenging, and so although there is simulation work no nucleation rates have been calculated. We discuss simulation work below, see 
Figure 5: SEM images obtained by Rodriguez-Blanco et al. ${ }^{48}$ of the phases which form during the two stages of the crystallization of calcium carbonate. The images in the top row are from the first stage. They show: (a) amorphous calcium carbonate (ACC); (b, c) ACC and vaterite nanoaggregates; and $(\mathrm{d}, \mathrm{e})$ vaterite nanoaggregates. Note that the vaterite forms as globular (see (e)) aggregates of nano-crystals of vaterite (see (d)). Images in the bottom row are from the second stage: (f) vaterite nanoaggregates and the first calcite crystals; (g) calcite crystals attached to vaterite spheres with the development of growth steps on the calcite surface; (h) calcite growth steps and vaterite nanoparticulate subunits; and (i) calcite crystals with vaterite casts. Reprinted from Ref. 48 with permission of The Royal Society of Chemistry.



also Raiteri et al. ${ }^{46,47}$ for recent work on calcium carbonate in aqueous solution, and a detailed discussion of the difficulties of developing a model of this system. Note that even modelling pure water is difficult, see the ice section, and that adding strongly interacting ions obviously makes the situation worse. In addition as we will see experiments suggest that there are dynamics with timescales that are far too long to be accessible to simulation.

Before we consider the recent experimental work on calcium carbonate crystallisation in solution we need to consider a rather puzzling state of calcium carbonate in solution that is presumably highly relevant to its crystallisation behaviour. This state is non-crystalline, it is in addition to the three crystal polymorphs: calcite, vaterite and aragonite. Crystals of two of the polymorphs of calcium carbonate, vaterite and calcite, are shown in Fig. 5.

\section{Amorphous Calcium Carbonate (ACC)}

There is extensive evidence that a concentrated non-crystalline phase can form in calcium carbonate solutions. ${ }^{48-54}$. This is called Amorphous Calcium Carbonate (ACC), see Fig. 5a for an SEM image of ACC. However, it may be misleading to call ACC a thermodynamic phase as apparently it can vary according to how it is prepared ${ }^{48-52}$. In particular, there is evidence for amorphous calcium carbonates with compositions ranging from almost no water to more than one water molecule per $\mathrm{CaCO}_{3}$ unit. This is inconsistent with a thermodynamic phase which (at a given $\mathrm{pH}$ and temperature) should have a definite composition when it coexists with the dilute solution.

The water dynamics in ACC with one water molecule per $\mathrm{CaCO}_{3}$ has been studied by Michel et $a l .{ }^{49}$, who found that some water in the ACC had rearrangement dynamics on the very slow timescale of milliseconds. Thus there are very slow dynamics in ACC, and at least for some compositions it may be close to being a glass.. Very slow dynamics offer a potential explanation for ACC's variability. Slow dynamics will inhibit the process of relaxing from the composition ACC is prepared in, to the 
equilibrium composition.

The slow dynamics, and it appears often time-dependent composition of ACC make understanding nucleation of a crystal phase in ACC very difficult. If the composition is changing then both the speed of the microscopic kinetics and the free energy barrier to crystallisation may be changing in a complex, uncontrolled and poorly understood way with time for a single sample, and it may be very different in samples prepared in different ways. Until we have much better quantitative data not just on the structure, but also on the microscopic kinetics in ACC, understanding crystallisation in ACC will probably be impossible.

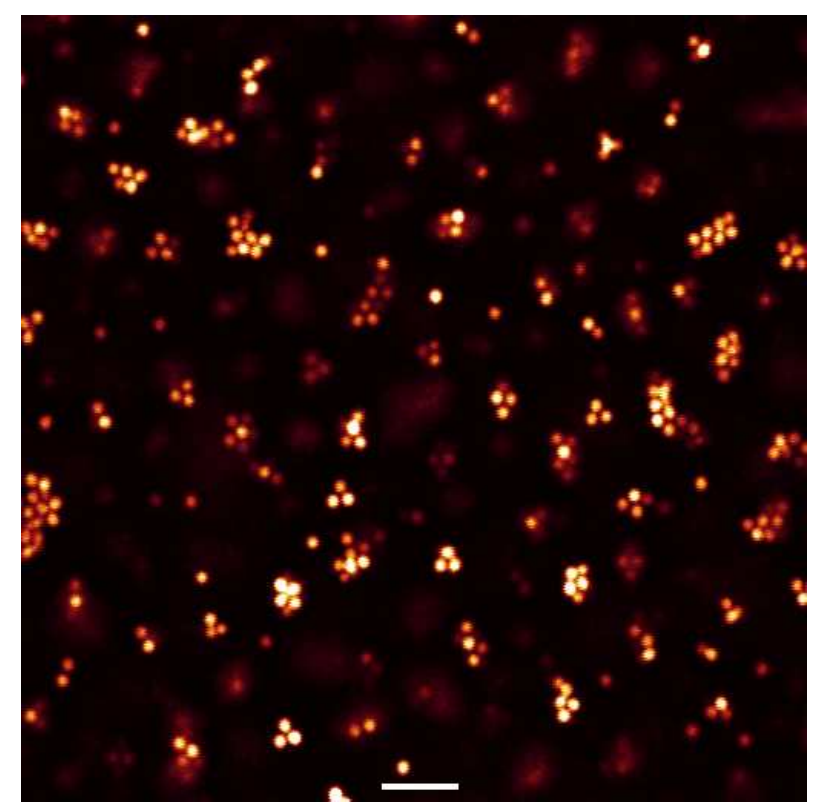

Figure 6: Clusters of colloidal particles, imaged with a confocal microscope ${ }^{55}$. The scale bar is $10 \mu \mathrm{m}$. The particles attract each other with a short-ranged attraction due to the presence of a non-adsorbing polymer in the suspension. The range of the attraction is set by the polymer size, which is 0.22 times the colloid diameter of $2 \mu \mathrm{m}$. They are also charged and so repel each other with a longer-ranged repulsion ${ }^{55}$. With this short attraction range the dilute-suspension/concentratedsuspension transition is within dilute-suspension/crystal coexistence. The attraction has a well depth of approximately $15 k T$ and the colloid volume fraction is 0.02 . The system is within both dilute-suspension/crystal and dilute-suspension/concentrated-suspension coexistence. The clusters are stable and do not coarsen into large crystallites over a period of days. Figure courtesy of C. P. Royall, see Ref. 55 for further details of the system.

\section{Nanoscale clusters of calcium carbonate in solution}

Now we return to consider recent experiments on nucleation in calcium carbonate solutions. Gebauer et al. ${ }^{56,57}$ find evidence of very long lived (minutes + ) clusters. The clusters are around $2 \mathrm{~nm}$ across, and so are of perhaps 70 calcium and carbonate ions. The clusters are present in both undersaturated and supersaturated solutions, and have been found by other workers ${ }^{58,59}$. Gebauer et al. also show that the solutions are strongly non-ideal. The experiments were done at high $\mathrm{pH}$. Gebauer et $a l$. propose that these clusters play a role in nucleation, and that nucleation is then non-classical in the sense that the nucleus does not grow single ion by single ion, but by the coalescence of clusters of many ions.

This is an interesting idea. Nucleation via the coalescence of clusters of tens of ions would be qualitatively different to classical nucleation theory's assumption of growth one ion at a time.

Unfortunately, evaluating this idea for calcium carbonate solutions is hindered by the fact that 
we do not know what the clusters are. Larger high density calcium carbonate aggregates can be studied via TEM, see below, but Gebauer et al. could not study the structure of their clusters. Clusters that are stable over relatively long timescales can be observed in the very different system of colloidal particles, see Fig. 6. There the clusters have crystalline local ordering and are stable for long times due to very slow kinetics. This may be what stabilises the calcium carbonate clusters. But it may also be a very different mechanism.

There are two obvious reasons for small clusters that persist for long times. The first is that the kinetics may be arrested, preventing both growth/coarsening of the clusters, and shrinking. This is the mechanism that stabilises the clusters in the colloidal system in Fig. 6. The second is that clusters are actually at least a local minimum in the free energy, i.e., increasing or decreasing the cluster does not occur because it would increase the free energy. In this section I will deal with these two mechanisms in turn. Note that here I will be neglecting any effects of a crystal lattice in the clusters. This neglect is harmless if the clusters are amorphous or fluid. However, the clusters may be crystalline. For the possible effects of a crystalline lattice, including mechanisms for arresting cluster growth, see the section on defects.

\section{Glassy clusters}

If the small clusters are not local free energy minima then something must be inhibiting their growth. Growth of clusters can occur either via pairs of clusters colliding and then coalescing, or via molecules leaving a smaller cluster and then diffusing to a larger cluster. This behaviour is discussed in any review on phase transition kinetics, such as that of Bray ${ }^{60}$. To stop growth both mechanisms need to be inhibited.

Coalescence will be slowed if the particles are either glassy or crystalline. However, standard theories of sintering e.g., via surface diffusion, predict that coalescence should be relatively rapid for small particles and only slow for large ones. The prediction is that the coalescence time $\tau_{c} \approx$ $R^{4} k T /\left(\gamma D_{s} a^{4}\right)$, where $R$ is the radius, $\gamma$ is the cluster surface tension, $D_{s}$ is the surface diffusion constant and $a$ is the molecular size. Picking a surface tension $\gamma=10 k T / a^{2}$ and a $D_{s}=a^{2} / \tau_{\text {hop }}$, where $\tau_{\text {hop }}$ is the time between hops of a molecule on the surface, we have that $\tau_{c}=\left(10 R^{4} / a^{4}\right) \tau_{\text {hop }}$. Then if $R$ is only few times $a$ the coalescence time is perhaps only 100 times the hopping time, and so coalescence is rapid unless surface diffusion is essentially completely arrested. This is supported by the simulations of Lewis et al. ${ }^{61}$, who find that their crystalline clusters do stick together and coalesce. This is in computer simulations in a model of gold but nanoscale calcium carbonate crystals should also tend to coalesce when they touch unless the dynamics are essentially arrested.

If clusters have more of one charged species than the other then they will have a net charge. A sufficiently large net charge will cause them to repel each other and hence stabilise them by dramatically reducing the rate at which their surfaces touch. However, quite large charges would be needed to prevent clusters touching over long periods.

Even if the clusters do not coalesce, perhaps because they are highly charged and so repel each other, growth can still occur via diffusion of molecules out of small clusters, across the intervening solution, and into larger clusters. This coarsening results in the typical cluster radius growing as $R(t)=\left(D_{\text {sol }} \gamma c_{\text {sol }} a^{3} / k T\right)^{1 / 360}$, where $D_{\text {sol }}$ and $c_{\text {sol }}$ are the diffusion constant and the concentration in the solution, respectively. This holds if the clusters exchange ions with the surrounding solution so that each cluster is in local equilibrium with the surrounding solution. This will be the case unless the cluster is glassy. Any reasonable value for $D_{\text {sol }}$ will give cluster radii that grow rapidly beyond sizes of a few nanometres. Clusters will only remain a few nanometres across if this mechanism does not operate, which would require that the clusters do not exchange ions with the surrounding solution and so are not in equilibrium with the solution.

A final point is that Gebauer et al. ${ }^{56}$ observe clusters in undersaturated solutions. In undersaturated solutions it is difficult to see why clusters would form unless the potential is such that the clusters are favoured even when a bulk concentrated phase is not, or the clusters are stabilised by impurities. We now discuss both these possibilities. 


\section{Clusters that are local free energy minima}

Normally, clusters whose dynamics are not arrested dissolve in an undersaturated solution and grow in a supersaturated solution. In a supersaturated solution nanoscale clusters should not persist as many small clusters have a much higher surface free energy penalty than a few larger ones and so the free energy is reduced by coarsening of many small clusters into a few large ones.

However, it is possible to have nanoscale clusters as a local free energy minima. Two obvious mechanisms for this are impurities and charge. It is well understood that impurities can stabilise nanoscale fluid clusters, at both under- and supersaturated conditions ${ }^{12-14,62,63}$. Here an impurity that strongly partitions into the phase that forms the clusters (ACC here?) stabilises the clusters.

The other mechanism is charge, this has been considered in the context of the nucleation of proteins (but not calcium carbonate) from solution by Hutchens and Wang ${ }^{64}$. Note that charge can generically stabilise mesoscale nanoscale objects and so essentially the same effect has been studied in a number of other contexts, e.g., for polyelectrolytes ${ }^{65}$. For calcium carbonate, if either calcium ions or carbonate ions partition more strongly than the other ion from water into the cluster, then the cluster will acquire a net charge. If the net charge density is $\rho_{Q}$ then there is then a Coulomb self-energy that scales as $\rho_{Q}^{2} v^{2} /\left(4 \pi \epsilon v^{1 / 3}\right)=\rho_{Q}^{2} v^{5 / 3} /(4 \pi \epsilon)$. This increases faster with cluster volume $v$, than either the surface free energy term, which scales as $v^{2 / 3}$, or the bulk driving force for crystallisation which scales as $v$. Hutchens and Wang ${ }^{64}$ show that this can create finite clusters that can be metastable, i.e., are at a local minimum in the free energy. For this to happen, one requirement is that they can be no larger than around the Debye length. The experiments of Gebauer et al. ${ }^{56}$ were performed at concentrations of the calcium and carbonate ions of around $10^{-4} \mathrm{M}$ but at high $\mathrm{pH}$, around 9 and above, where the concentration of $\mathrm{OH}^{-}$ions is around $10^{-2} \mathrm{M}$. Thus the Debye length is around a few nanometres, and so the clusters are not too large to be charge stabilised.

In summary, unless the clusters have large charges, are stabilised by impurities, or are kinetically arrested, it is hard to understand their stability. It is also not clear how they contribute to nucleation, indeed we have not direct proof that they do so. The observations of Gebauer et al. ${ }^{56}$ are intriguing but we are clearly very far from having even a qualitative picture of how calcite is nucleating in this system. More experimental data is needed.

One possible experimental test could be to quantify how the cluster concentration varies with concentration. In a dilute solution, any clusters will be weakly interacting and so the density of pure clusters of $n$ molecules is expected to vary very rapidly, as $c_{M}^{n}$, for $c_{M}$ the monomer concentration. This is for clusters at a local free-energy minimum, which are not stabilised by impurities. The concentration of clusters that are stabilised by glassy dynamics should generally vary less rapidly and the concentration of clusters stabilised by impurities would vary with impurity concentration.

\section{Larger calcium carbonate aggregates}

Nudelman et al. ${ }^{59}$ studied rather larger calcium carbonate aggregates, $10 \mathrm{~nm}$ and larger. They observed that their aggregates are ACC not crystalline for sizes less than approximately $100 \mathrm{~nm}$, but are crystalline at larger sizes. This is surprising. The surface term in the free energy can mean that small clusters can be fluid at equilibrium even when in the bulk the equilibrium phase is crystalline, but this is only true of very small clusters. I will discuss why below, then consider whether the kinetics of freezing can explain small clusters being amorphous and larger ones crystalline.

Small clusters can be fluid at equilibrium even when in the bulk the equilibrium phase is crystalline. This will be the case if the surface tension of the fluid phase is lower than that of the crystal phase. A lower surface tension stabilises clusters in the fluid state below a cross-over radius of approximately $R_{X} \simeq \Delta \gamma v_{C C} /(\Delta \mu)$. Here $\Delta \gamma$ is the difference between the surface tension of calcite in water and the surface tension of ACC in water, and $\Delta \mu$ is the difference between the chemical potentials of the two phases. $v_{C C}$ is the volume of one calcium carbonate species. Given that $\Delta \mu$ is not much less than $k T$ and that $\Delta \gamma$ is unlikely to be more than a few $k T$, this gives radii that are at most a few times the effective diameter of calcium carbonate. This is a few nm, not $100 \mathrm{~nm}$. 
Here, by stabilise we mean reduce the free energy of the nanoparticle of the less stable phase such that it is lower than the free energy of a nanoparticle of the same size of the equilibrium phase. See van Meel et al. ${ }^{66}$ for a discussion of this for the Lennard-Jones system.

Simple kinetics can explain a sharp crossover from small clusters that have not crystallised to larger clusters that have crystallised, but only during a time window. The argument is as follows. The probability that the crystal phase has not nucleated in a cluster and so is still amorphous is $p_{a}(R)=\exp \left(-r_{x}(R) t\right)$, where $r_{x}(R)$ is the nucleation rate in a cluster of radius $R$. If nucleation occurs at the surface - here the nucleation barrier should be lower and the microscopic kinetics faster - then $r_{x}$ should scale as $R^{2}$. Then if $p_{a}(R=50 \mathrm{~nm})=0.01, p_{a}(R=5 \mathrm{~nm})=0.95$; it is easy to see that is possible to have most small clusters still ACC while the large ones are almost all crystalline, simply due to a larger nucleation rate in the larger clusters. Note that this scaling assumes a uniform nucleation rate on the surface, if nucleation is occuring on an impurity at the surface this may not be true.

However, Nudelman et al. ${ }^{59}$ found that the ACC clusters were stable for long periods. A kinetic explanation cannot explain this as although reducing the diameter by a factor of ten will stabilise the cluster for 100 times as long, eventually it will crystallise. This failure of both an equilibrium argument and simple kinetic argument leaves us with more questions than answers.

(A)

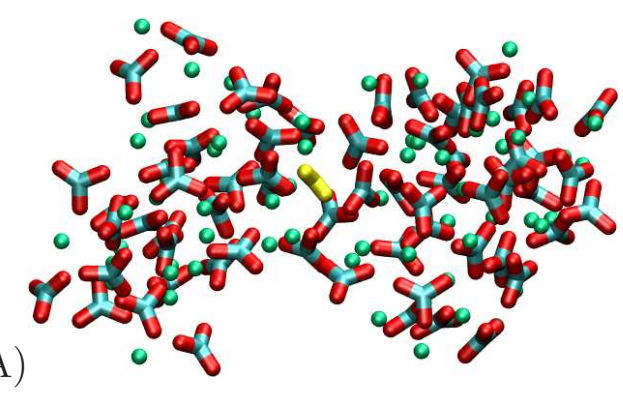

(B)

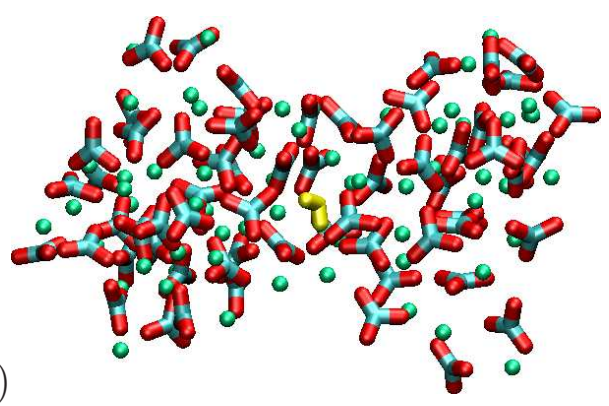

Figure 7: Two snapshots 0.58 ns apart, from simulations by Tribello et al. ${ }^{67}$ of a model of calcium carbonate in solution. The calcium ions are shown as green spheres, the carbonate ions in cyan and red, and a single water molecule is shown in yellow. In (A) the water molecule is at the surface but between two ACC clusters. In (B) it is surrounded and trapped by calcium and carbonate ions. (B) is 0.58 ns later than (A). Reprinted from Ref. 67 with permission. Copyright 2009 American Chemical Society.

\section{Computer simulation of calcium carbonate}

The study of the crystallisation of calcium carbonate from solution poses formidable challenges to computer simulation. The solutions in experiment are dilute (therefore many water molecules must be simulated in addition to the calcium and carbonate ions), the ions are divalent and so interact strongly, and there is an intermediate ACC phase with what experiment ${ }^{49}$ suggests are slow dynamics - much slower than the timescales that are accessible in a direct computer simulation. These problems have prevented any direct study of the complete process of nucleation from solution. However, calcium carbonate is a system of great importance and there is an urgent need for information on the microscopic dynamics which only simulation can provide. So a number of studies have been made, and valuable results have been obtained ${ }^{46,47,67,68}$.

Tribello et al. ${ }^{67}$ found that in their highly supersaturated solutions, ACC grew much more rapidly than calcite. At least at high supersaturations, it is possible that ACC could form instead of the more stable calcite simply because ACC can out-compete the calcite by growing faster. They also found that water molecules can be trapped when two amorphous calcium carbonate clusters coalesce, see Fig. 7. Water molecules being trapped in growing ACC clusters would be consistent with the water content of ACC being variable, as the amount of water would then be determined by 
the kinetics of growth. However, it should be borne in mind that the simulation runs were of $20 \mathrm{~ns}$ duration or less, which is many orders of magnitude smaller than the ms timescales found for some water in ACC by Michel et al. ${ }^{49}$. Also, as with all other simulations it should be remembered that a simple potential is used, which may not reproduce all the behaviour of the calcium carbonate in water system correctly. See the recent work of Raiteri and coworkers for a discussion of potentials for calcium carbonate in water ${ }^{46,47}$.

Quigley and coworkers ${ }^{68,69}$ simulated clusters with diameters from around 2 to $5 \mathrm{~nm}$. In their second, more thorough study ${ }^{69}$, they found that their cluster had a lower free energy as a calcite nanoparticle than as an (anhydrous) amorphous cluster. This suggests that small ACC clusters are not stabilised by having a lower surface tension than the crystal. If ACC nanoclusters are not stabilised by a relatively low surface tension then that would suggest that they are only favoured by kinetics. ACC may grow faster from solution than calcite, and then persist due the large nucleation barrier to crystallisation in ACC found by Quigley et al. ${ }^{69}$. However, this prediction is for amorphous calcium carbonate without water, and it be may sensitive to the potential used.

\section{Overview of nucleation that overcomes more than one barrier or is associated with a different phase transition}

This and the remaining sections of the review are thematic, each one reviews a particular cause of non-classical nucleation behaviour, such as an intermediate phase. This cause may occur in many different systems, and so each of the remaining sections may contain experiments and simulation results. I hope that those scientists who think that their crystals may be nucleating via a nonclassical pathway can find a section or sections here that describe the behaviour they observe, and hence learn of other work studying related behaviour.

Consider the following common situation. A scientist is studying a supersaturated solution (or melt or vapour ...). Call this solution phase A. They cool it so the solution is supersaturated with respect to a crystal phase B. This crystal is then the equilibrium phase. At some small supercooling, they see nothing happening. Clearly there must be at least one large nucleation barrier between the supersaturated phase A and the crystal phase B. In principle, there could of course be more than one large nucleation barrier along paths from A to B, and there could be multiple competing paths between $\mathrm{A}$ and $\mathrm{B}$.

If on further cooling, the crystal phase B nucleates rapidly then clearly there is now a pathway between the supersaturated phase and the nucleated phase, along which there are no large barriers. However, this pathway does not have to be simply a nucleus of B forming in A. As Ostwald understood more than 100 years ago, a third phase, call it phase $\mathrm{C}$, could intervene. With a phase $\mathrm{C}$, the pathway from A to B could be as follows: $\mathrm{C}$ nucleates in A, then B nucleates in or on the surface of C. In this two-step pathway, either of the steps could be slow activated processes.

It is unlikely that both barriers will be the same, and so one will be larger and hence rate limiting. For example, if there is a large nucleation barrier to the nucleation of $\mathrm{C}$ in $\mathrm{A}$, but the nucleation of $\mathrm{B}$ in $\mathrm{C}$ is rapid, then the rate-limiting step will be the nucleation of $\mathrm{C}$. Thus, here the rate-limiting nucleation process is of a transient phase that may never appear in amounts large enough to be observed. Understanding that the rate is set by the nucleation of a transient phase that is not B, would be crucial to understanding the kinetics here ${ }^{70,71}$. Assuming that the rate limiting step is the nucleation of the final phase, phase B, will lead to the wrong conclusions being drawn.

Particularly if the two barriers are not too different then the two-step nature of the kinetics may show up via deviations from the Kolmogorov-Johnson-Mehl-Avrami (KJMA) rule for the dependence of the fraction crystallised as a function of time, see the model of Kashchiev and Sato ${ }^{72}$, and the model and data of both Morales-Sánchez et al. ${ }^{73}$ and Laine et al. ${ }^{74}$. This data is for alloys of germanium, antimony and tellurium.

Nucleation is probably occurring on the surface of an impurity, or of the container. Thus, we have slow nucleation of $\mathrm{C}$ at a surface, followed by rapid nucleation of $\mathrm{B}$ in the new phase $\mathrm{C}$. Now, 


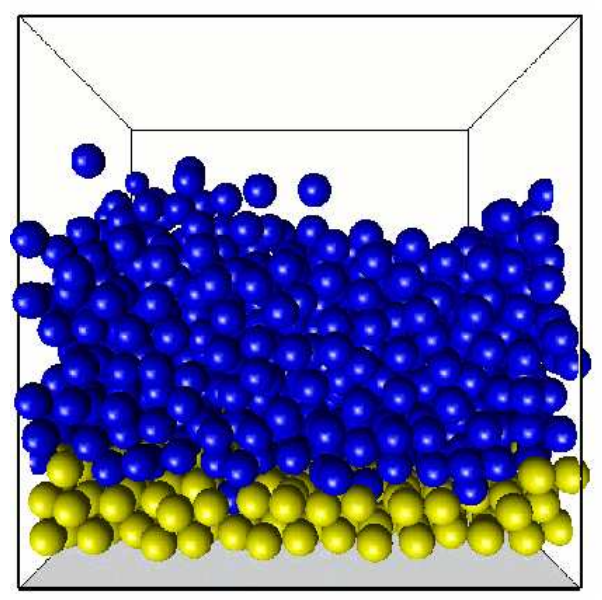

Figure 8: A simulation snapshot showing a surface (light grey plane at the bottom of the simulation box) on which the surface transition pre-freezing has occurred, from Page and Sear ${ }^{76}$. The system is above the triple-point temperature, so the crystal is not stable in the bulk at the low pressure of the simulation. Instead in the bulk there is vapour/liquid coexistence. The yellow particles are Lennard-Jones particles in a locally crystalline environment while the blue particles are the same Lennard-Jones particles but in locally fluid environments. Note that approximately two layers of particles have crystallised at the surface but that the bulk is still liquid. This freezing at the surface is the surface phase transition called pre-freezing. Adapted from Ref. 76 with permission. Copyright 2009 by the American Physical Society.

it is well understood that there can be a rich and complex phase behaviour at surfaces, with its own hysteresis. See for example the book of Butt, Grad and Kappl ${ }^{75}$ for an introduction to interfaces.

Thus, at a solid surface, instead of a new bulk phase $\mathrm{C}$ appearing we could have a surface phase transition, for an example see Fig. 8. For instance, we could have a situation where there are two surface phases, call them $\alpha$ and $\beta$. The rate of heterogeneous nucleation of the new bulk crystal $\mathrm{B}$ could be much higher in surface phase $\beta$ than in surface phase $\alpha$. Then the rate limiting step for the formation of bulk phase B from bulk phase A could be the formation of the surface phase $\beta$. For an example of this, see Page and Sear ${ }^{76}$. Interpreting experimental data correctly will then require detecting and understanding the $\alpha$-to- $\beta$ surface phase transition. This will often be difficult, particularly if it is occurring on a nano-scale impurity particle.

As there are so many possibilities for nucleation that involve another bulk or a surface phase transition, we have split up this part of the review into several sections. The first section is this one, and it just introduces the basic idea. The next section is devoted to the nucleation of one crystal phase being accelerated by a second bulk crystal phase. Subsequent sections review systems where a metastable fluid phase appears to affect the nucleation of the crystal. There is also a section towards the end of this review that cover nucleation in wedges and pits. See that section for a discussion on how nucleation of a crystal phase in pits can be affected by capillary condensation near a vapour/liquid transition. Capillary condensation is a surface phase transition.

\section{Crystallisation in the presence of multiple polymorphs}

Many substances have more than one polymorph, for example the substance generally called ROY has seven polymorphs with known crystal structures ${ }^{78,79}$. Indeed this substance's common name of ROY comes from the Red, Orange and Yellow crystal polymorphs it forms. For substances with more than one polymorph, we have no way of predicting which polymorph will appear first, i.e., will apparently nucleate fastest, or which one will grow fastest. Over one hundred years ago Ostwald 
Figure 9: Optical micrographs showing: a) the selective formation of the anhydrous form of theophylline on a carboxy-terminated Self-Assembled Monolayer (SAM), and b) the selective formation of the monohydrate form on a different SAM, a methyl-terminated SAM. This is from the work of Cox et al. ${ }^{77}$. Under the conditions of the experiments the monohydrate polymorph is more stable than the anhydrous. In both cases nucleation was presumably heterogeneous and occurred on the SAM. Reprinted from Ref. 77 with permission. Copyright Wiley-VCH Verlag GmbH \& Co. KGaA.
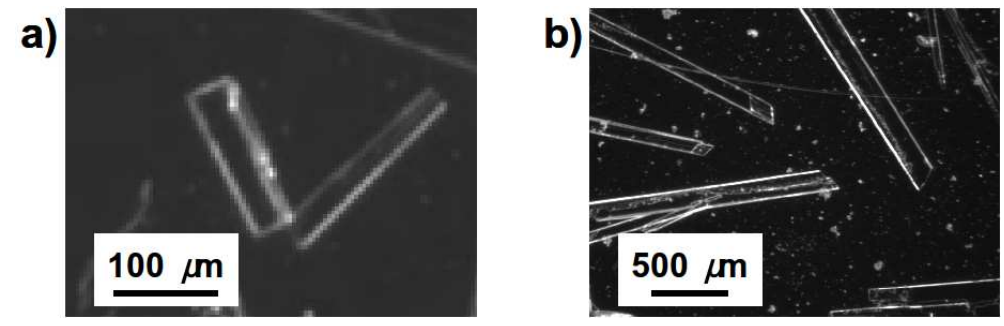

postulated that it is not the equilibrium phase that nucleates first, but the phase that is closest in free energy to the starting phase, i.e., when there is a phase with a free energy intermediate between the starting phase and the equilibrium phase, it is this intermediate phase that nucleates. ${ }^{80,81}$. This is not always true, see the example in Fig. 9, but it does highlight the fact that we cannot assume it will be the equilibrium phase that nucleates. In Fig. 9, on one Self-Assembled Monolayer (SAM) the less stable crystal polymorph forms (the anhydrous form, Fig. 9a) as Ostwald's rule would predict, but on a different SAM a more stable polymorph apparently nucleates first the monohydrate, Fig. 9b) ${ }^{77}$. This illustrates the observation that Ostwald's rule is only true some of the time, and that the surfaces present can control (presumably via heterogeneous nucleation) the polymorph that appears. Also, traces of impurities can alter the polymorph that forms, see Fig. 2.

$\mathrm{Yu}$ and coworkers have made some particularly interesting studies of the nucleation and growth of competing polymorphs, in molecular solids. Yu studied D-mannitol in $2003^{82}$. D-mannitol has 3 polymorphs, in order of increasing stability under the experimental conditions, these are $\delta, \alpha$ and $\beta$. In the 2003 experiments $\mathrm{Yu}$ did not observe the most stable $\beta$ form. He observed that the $\delta$ polymorph formed first and then it appeared that the more stable $\alpha$ polymorph nucleated on crystals of the $\delta$ polymorph and then essentially outgrew the less stable polymorph. Similar behaviour was observed for ROY ${ }^{83}$. Given that the initial nucleation of the $\delta$ polymorph was probably on an impurity, it appears that the nucleation steps were heterogeneous nucleation of the $\delta$ polymorph on an impurity followed by nucleation of the $\alpha$ polymorph on a surface of a crystal of the $\delta$ polymorph.

Later work with Tao and Jones on seeded nucleation with $\beta$ seeds found that the less stable $\alpha$ polymorph could nucleate (at larger supercoolings) on the more stable $\beta$ crystals, and then grew faster than the $\beta$ form. An $\alpha$ crystal that has nucleated on a $\beta$ seed is shown in Fig. 10.

$\mathrm{Yu}$ also studied D-sorbitol, which has the polymorphs E, A, B and $\gamma$, in order of increasing stability under the conditions of the experiment. There he found only the E polymorph. This polymorph was the only one that formed both with and without seeding with the stable $\gamma$ polymorph. This suggests ${ }^{82}$ that under the experimental conditions, the E polymorph has a lower nucleation barrier than the $\gamma$ polymorph, and that it grows faster.

This phenomenon of one polymorph nucleating and growing, but then being overtaken by another polymorph that nucleates on the surface of the first polymorph, is common. See Refs. 79,83-85 for other examples. As the nucleation of the first polymorph is presumably usually heterogeneous, and may occur on the surfaces of the container, then altering the surface chemistry of these surfaces is one way to control the polymorph, see Fig. 9 and the work of Cox et al. ${ }^{77}$.

Sirota and Herhold ${ }^{70}$ studied nucleation of the stable polymorph that is accelerated by the appearance of a transient and less stable polymorph. Transient here means that it is difficult or impossible to stabilise large volumes of it for long times. Their experiments were on alkanes where the stable phase is a triclinic crystal, and the transient phase is a rotator crystal phase. 
Figure 10: Crystallisation of D-mannitol from the liquid phase, when a seed of the $\beta$ polymorph is added to the liquid, from Tao et al. ${ }^{87}$. The $\beta$ polymorph is more stable than the $\alpha$ polymorph, and at the start the $\beta$ polymorph is already present as a seed crystal. However the $\alpha$ form nucleates on the surface of the $\beta$ polymorph. Then despite its lower stability, the $\alpha$ polymorph has a higher growth rate than the $\beta$ polymorph, and so can out-grow the $\beta$ polymorph. Reprinted from Ref. 87 with permission. Copyright 2007 American Chemical Society.

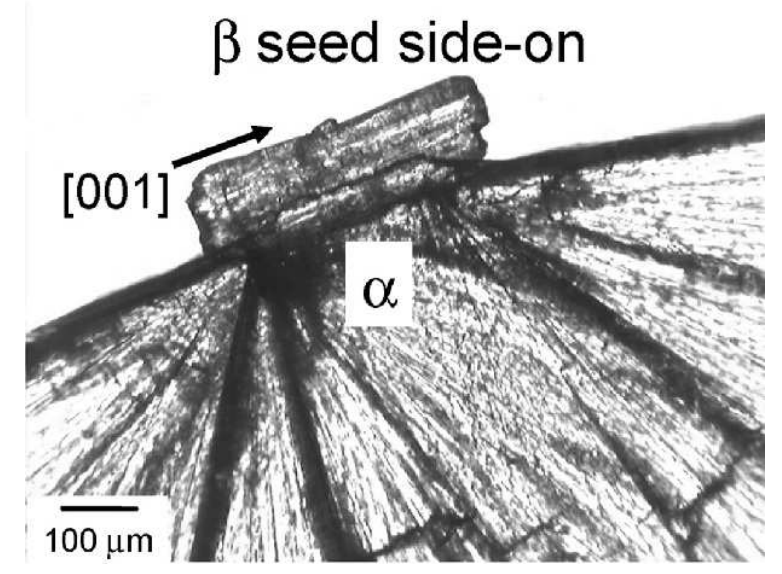

Also, Hughes and Harris ${ }^{71}$ have shown how to use NMR to follow a transient polymorph as it forms and then rapidly transforms to a more stable polymorph. They studied solutions of glycine crystallising from methanol/water mixtures, and found that the $\beta$ polymorph of glycine formed rapidly but then the amount of this form dropped as the amount of the more stable $\alpha$ polymorph increased. The transformation is probably via dissolution of the $\beta$ polymorph occuring in parallel with crystallisation of the $\alpha$ polymorph from the solution that surrounds both crystals. In other words it is a recrystallisation process not a direct solid-to-solid process.

One polymorph can also transform to another in the solid state. Here nucleation is likely to be occurring at a defect in the solid ${ }^{2}$. An interesting example of this is found in chocolate ${ }^{86}$. The white bloom found on chocolate stored at high temperatures is due to a transformation to a more stable polymorph of a constituent of chocolate. The new polymorph has a different unit cell so the transformation causes strain to build which is released by the formation of micrometre and smaller flakes of the new polymorph. These flakes are responsible for the white bloom ${ }^{86}$.

So far we have only considered molecular systems, similar phenomena can occur in ionic systems. For example, the most stable polymorph of calcium carbonate, calcite, can form via a less stable polymorph, vaterite. See for example the recent work of Rodriguez-Blanco et al. ${ }^{48}$. In common with other experimental work, Rodriguez-Blanco et al. cannot observe nucleation. However the large number of nanocrystals of vaterite suggest a high nucleation rate. The arguments of section on nucleation at vapour/liquid interface suggest that it is likely that this nucleation occurs on the surface of the amorphous calcium carbonate (see that section and the section on calcium carbonate section) but this suggestion has not been tested. Vaterite was observed to be fully formed within 45 minutes, at $7.5^{\circ} \mathrm{C}$. The conversion to calcite then took around 18 hours. The crystals of calcite are orders of magnitude bigger, micrometres across not tens of nanometres. As they are perhaps 100 times bigger, see Fig. 5(i), but take 30 times as long to form it appears that the growth rates (under the conditions each grows, which are presumably different) are quite similar. This suggests that vaterite may be appearing not because it grows much faster than the more stable calcite form but because its nucleation rate is much higher.

This work is an interesting example of a large amount of recent work, there is extensive literature on the crystallisation of calcium carbonate, much of inspired by the fact that living organisms such as 
sea urchins, molluscs, etc., have an impressive ability to control which calcium carbonate polymorph forms. See the review of Meldrum and Cöfen ${ }^{44}$, and the books of Cölfen and Antonietti ${ }^{43}$, and Mann ${ }^{45}$, for introductions to this literature.

\section{Simulation}

Desgranges and Delhommelle ${ }^{88-90}$ have used computer simulations to study models in which the relative stability of the fcc and bcc polymorphs can be varied. They studied homogeneous nucleation in a simple model at relatively large supercoolings, so the system is rather different from the typical experimental situation. However, simulation has the advantage over experiment of being able to see the nucleus overcoming the nucleation barrier, and it can follow the growing crystallite when it is still microscopic. Desgranges and Delhommelle studied nucleation and the early stages of growth, under conditions where the fcc phase was a little more stable than the bcc phase. They found ${ }^{88-90}$ that at small sizes the nucleus had a large bcc component but that as it grew, fcc took over. This occurred when the nucleus was still microscopic, i.e., far too small to be seen in an experiment.

This is an interesting result, in experiment one polymorph is often seen to nucleate on another, see for example Fig. 10. However this is when the first polymorph to form has grown to macroscopic dimensions and been observed, Tao et al. used optical microscopy. If for example, a less stable polymorph nucleates faster but grows more slowly than a more stable polymorph, then the less stable polymorph may only appear very transiently at the nucleation step, before the more stable polymorph nucleates on it and then out-grows it. Then this less stable polymorph may play a key role in nucleation but exist for only a small fraction of second and in the form of a crystallite of perhaps only a hundred or a thousand molecules. This will not be observed in experiment. Thus, "hidden" transient polymorphs could easily be playing an unappreciated role in nucleation, and not appreciating their role could be leading to errors. For example, if nucleation is epitaxial then to encourage nucleation a substrate with a good epitaxial match to this transient polymorph may be more effective than one with a good epitaxial match to the polymorph actually observed.

Browning et al. ${ }^{91}$ also used computer simulation to study a simple model with bcc and fcc polymorphs. They introduced seed crystals of the less stable polymorph into supercooled fluids, and looked for whether the more stable polymorph would nucleate. They introduced a bcc seed crystal into the fluid at low supersaturations with respect to the equilibrium fcc crystal, and found that the bcc seed crystal would continue to grow, without the more stable fcc crystal nucleating. Under the same conditions an fcc seed produced fcc crystallisation, as expected. Thus at low supersaturations the seed crystal controlled the phase that formed, as there nucleation of both the bcc and fcc phases was slow.

\section{Examples of crystallisation in the presence of a fluid/fluid transition}

Crystallisation can occur from a dilute phase, i.e., from a phase in which the molecules that will crystallise are present at a volume fraction of only a few $\%$ or less. This is typically either a vapour phase or a solution. Crystallisation then requires attractions between the molecules to pull them together into a crystal. These same attractions also tend to produce a fluid/fluid transition.

In the case of a vapour the fluid/fluid transition is a vapour/liquid transition while in solution it is a dilute-solution/concentrated-solution transition, also called a liquid/liquid transition, phase separation or "oiling out". For our purposes there is little real difference between any of these phase transitions. The result is always the same: a new phase in which the crystallising molecules are present at much higher concentration. This concentration is typically only a little below that in the crystal.

We expect that the interfacial tension between the crystal and the liquid phase is smaller than the interfacial tension between the crystal and the vapour phase. This is true for some simple systems such as the Lennard-Jones model ${ }^{66}$. Then classical nucleation theory predicts that the nucleation rate of crystallisation should be faster in a liquid than in the coexisting vapour, and so the presence 
of the liquid will accelerate crystallisation. Note that here we will mostly use "vapour" and "liquid" for simplicity but the reader can replace these with dilute and concentrated solution if they wish.

There are a number of experimental systems where there is evidence that an intermediate liquid or concentrated-solution phase plays a role in crystallisation from a vapour or from a dilute solution:

1. During attempts to crystallise them, solutions of many proteins often separate into dilute and concentrated solution phases. Most of the quantitative studies of this have worked with the small, stable cheap protein lysozyme ${ }^{11,92-95}$. Protein crystallisation is of great importance due to the need for protein crystals in order to use X-ray diffraction to determine their structure. However most proteins cannot be crystallised. As I discuss in my earlier review ${ }^{1}$ there is extensive evidence that this dilute-to-concentrated-solution strongly influences crystallisation in lysozyme.

2. There is also evidence that crystallisation of some relatively small molecules can also occur via the formation of a concentrated solution phase, e.g., Bonnett et al. ${ }^{96}$ observed liquid-liquid phase separation, also called oiling out in this field, followed by crystallisation in the molecule methyl(E)-2-[2-(6-trifluoromethylpyridine-2-yloxymethyl)-phenyl]-3-methoxyacrylate.

3. An amorphous phase ${ }^{43,44,49,50}$ frequently appears in supersaturated aqueous solutions of calcium carbonate, and clearly effects crystallisation, see Fig. 5. Similar phenomena may be occurring in other ionic systems ${ }^{42}$ although these are much less studied. See the calcium carbonate section.

4. Nucleation of the crystal phase in liquid metal mixtures is of interest in the preparation of metallic glasses. To make a glass nucleation must be sufficiently slow to allow the liquid mixture to be cooled down below the glass transition temperature before crystal nuclei can form ${ }^{97}$. Gebert et al. ${ }^{98}$ found that varying the composition of their metallic system $\mathrm{Zr}_{65} \mathrm{Al}_{7.5} \mathrm{Cu}_{17.5} \mathrm{Ni}_{10}$ (by increasing the oxygen content) caused a metastable fcc $\mathrm{NiZr}_{2}$ phase to appear which in turn lead to the formation of the stable crystals $\mathrm{CuZr}_{2}$ and $\mathrm{NiAl}_{2} \mathrm{Zr}_{6}$.

5. In the Earth's atmosphere ice can nucleate from cold $\left(<0^{\circ} \mathrm{C}\right)$ wet air, to form snow. At the temperatures involved, say around -5 to $-30^{\circ} \mathrm{C}^{12}$, water is only marginally (a few tenths of a $k T$ ) less stable than ice, and so at all but small supersaturations the air is either close to or actually supersaturated with respect to liquid water, as well as being supersaturated with respect to ice. Often droplets of water form and then freeze ${ }^{12}$. This is discussed in the ice section.

6. In colloidal systems the particles can be directly imaged and followed. Zhang and $\mathrm{Liu}^{99}$, and Savage and Dinsmore ${ }^{100}$ have taken advantage of this and directly imaged transient dense amorphous clusters that form in a dilute suspension, and then crystallise.

Note that in some cases the liquid or concentrated solution phase that forms may be highly viscous or even glassy. For example, there is evidence of slow water dynamics in the amorphous phase of calcium carbonate ${ }^{49}$, and the concentrated solution phase of lysozyme and other proteins is under at least some conditions gel-like ${ }^{101}$. High viscosity will slow the nucleation and growth of a crystal inside a concentrated phase. If the dynamics arrest completely then nucleation will be prevented, even if the nucleation barrier is low.

A liquid or concentrated fluid phase is closer in density to a crystal phase than a dilute vapour or solution, and this closeness is generally expected to reduce the nucleating crystal's surface tension and hence facilitate nucleation. However, by definition a liquid phase has none of orientational and translational ordering of a crystalline phase. By contrast liquid crystalline phases are not only dense but also are partially ordered and so are even closer to crystal phases than liquids are. Janbon et al. ${ }^{102}$ studied crystallisation via a nematic phase. They suggest that crystallisation from a nematic phase may favour the crystal polymorph whose ordering is closest to that in the nematic phase. In 
the systems that form these liquid crystalline phases, they could potentially be even more effective than liquid phases at helping the nucleation of a crystal.

The nucleation in the experiments listed above is presumably heterogeneous and on impurity particles, but for clarity of presentation I will first outline possible mechanisms for how an intermediate liquid phase affects homogeneous nucleation. I will start with homogeneous nucleation via a liquid that is more stable than the vapour. Here macroscopic amounts of the liquid can form, and persist for long periods of time. Then I will discuss the situation where the liquid phase is a little less stable than the vapour phase. Then in the bulk, only a microscopic amount of liquid can form, and it can only exist transiently. However, only a microscopic amount of the liquid is enough to greatly accelerate nucleation of the crystal. Only enough liquid to accommodate a microscopic crystal nucleus is required, and it only has to persist long enough for the nucleus to cross the barrier. I will then consider nucleation at the vapour/liquid interface. Nucleation at surfaces and in pores is considered in two sections towards the end of this review.

\section{Nucleation of a crystal phase when the metastable liquid is more stable than the vapour}

Within classical nucleation theory the barrier for nucleation of phase $\alpha$ in phase $\beta$ scales as $\gamma_{\alpha \beta}^{3} / \Delta \mu_{\alpha \beta}^{2}$. Here $\gamma_{\alpha \beta}$ is the interfacial tension between the nucleus of phase $\alpha$ and its surroundings of phase $\beta$. $\Delta \mu_{\alpha \beta}$ is the difference between the chemical potential of phase $\alpha$ and the chemical potential of phase $\beta$. Thus if

$$
\frac{\gamma_{x v}^{3}}{\Delta \mu_{x v}^{2}}>\frac{\gamma_{l v}^{3}}{\Delta \mu_{l v}^{2}}, \quad \frac{\gamma_{x l}^{3}}{\Delta \mu_{x l}^{2}}
$$

then nucleation should be faster in two steps, liquid from vapour then crystal from liquid. Here $\gamma_{x v}, \gamma_{l v}$ and $\gamma_{x l}$ are the interfacial tensions for the crystal/vapour, liquid/vapour and crystal/liquid interfaces, respectively. $\Delta \mu_{x v}, \Delta \mu_{l v}$ and $\Delta \mu_{x l}$ are the chemical potential differences for the crystal and vapour, liquid and vapour, and crystal and liquid pairs of phases, respectively.

If nucleation is in two steps then the slower rate will determine the rate. This means, for example, that use of the nucleation theorem ${ }^{1}$ should give an estimate of the number of excess number of molecules in the rate-limiting nucleus. Also, as $(\partial \mu / \partial p)=1 / \rho$ the chemical potential of dilute phases changes rapidly with pressure, $p$, while that of dense phases changes only slowly with pressure. Here $\rho$ is the number density of molecules. Thus as the pressure of a vapour or concentration of a solution, is varied, $\Delta \mu_{l v}$ rapidly increases while $\Delta \mu_{x l}$ remains almost unchanged. This means that if nucleation of the liquid phase is rate limiting, then the rate at which crystal nuclei form will rapidly increase with pressure. However, if the rate limiting process is nucleation of the crystal from the liquid, then the rate at which crystal nuclei appear may be almost independent of pressure $^{66}$. In solutions the same argument applies, if the rate increases rapidly with concentration or osmotic pressure then this suggests that nucleation of the concentrated phase is rate limiting.

Both Chen et al. ${ }^{103}$ and van Meel et al. ${ }^{66}$ used computer simulations of the Lennard-Jones model just below its triple point to study crystallisation from the dilute vapour. As expected from the above arguments, it is clear that there are conditions where homogeneous nucleation proceeds via two steps: nucleation of the liquid, followed by nucleation of the crystal in liquid droplets. Formation of a crystal directly in the vapour occurs at a much lower rate. The liquid phase, which is not present at equilibrium as we are below the triple point, greatly accelerates crystallisation. Duff and Peters ${ }^{104,105}$, and Whitelam ${ }^{106}$ have both used computer simulation to study simple lattice models in which the analogue of a vapour-liquid transition can provide a stepping stone to the system's ordered phase.

The general expectation is that the interfacial tensions will be in the order $\gamma_{x v}>\gamma_{l v}>\gamma_{x l}$. This is the case in the Lennard-Jones model ${ }^{66}$, but it does not always have to be true. Some solids, the Lennard-Jones model is an example ${ }^{66,107}$, surface melt. There it is true that $\gamma_{x v}>\gamma_{x l}+\gamma_{l v}$, and so when all 3 phases coexist at the triple point, there is a thick layer of the liquid phase between 
the crystal and the vapour. Interposing a liquid between the crystal and a vapour lowers the free energy. Ice also surface melts ${ }^{28-30}$, but some other substances do not, such as gold ${ }^{108,109}$.

In many systems the liquid phase accelerates crystallisation. However, there are circumstances where it can inhibit crystallisation. This can be for thermodynamic or kinetic reasons. If the liquid is only a little less stable than the crystal the driving force for crystallisation in the liquid, $\Delta \mu_{x l}$, will be small and so the nucleation barrier to crystallisation will be large. This is a general feature of two-step nucleation, if the intermediate phase (here the liquid) is not stable enough it cannot help nucleation but if it is too stable then it forms but then persists, i.e., the second step does not occur and so no crystals form. There is a window of relative stability where the intermediate phase helps nucleation ${ }^{110}$. Also, if the liquid phase is gel or glass-like, i.e., if diffusion of the molecules in it is very slow then its formation may also hinder crystallisation. The system may arrest in this glassy phase and then no crystals will form, even though the molecules may have been highly dynamic in the original vapour or dilute solution.

\section{Nucleation of a crystal phase when the metastable liquid is less stable than the vapour}

In 2000, Galkin and Vekilov ${ }^{93}$ found a maximum in the apparent rate of nucleation of lysozyme crystals just outside a dilute-to-concentrated-solution transition. Naively, you might expect a diluteto-concentrated-solution or vapour/liquid transition to only be able influence crystallisation when a system is within the transition. This is not the case, but it is true that outside vapour/liquid coexistence only microscopic amounts of liquid can form. This brings us to discuss the influence of microscopic droplets of the liquid phase. Note that we have already done so in the context of calcium carbonate, so there is overlap between the calcium-carbonate section and this section. Also note that the liquid phase here may be very viscous, possibly even glassy.

Most of the experimental studies of the effect of clusters of protein molecules have been undertaken on solutions of the small protein lysozyme. However, clusters have also been invoked in studies of crystallisation in systems of smaller molecules. For example, Chattopadhyay et al. ${ }^{111}$ observed clusters of a few molecules of glycine in supersaturated aqueous solutions. In this section, I will start by outlining how a "conventional liquid" (concentrated solution etc.) phase can aid nucleation, then outline the data for lysozyme and possible models for this behaviour. By "conventional liquid" I mean a liquid whose droplets have a free energy that can be written as a sum of a volume term and a (positive) surface term, and so do not not form clusters at equilibrium. See the calcium carbonate section for a discussion of nucleation in the presence of cluster-forming systems.

Outside vapour/liquid coexistence, the concentrated-solution phase is less stable than the dilute phase. Therefore, (without impurities) in the bulk a liquid droplet can only form transiently. So the liquid droplet can only aid homogeneous nucleation during its brief lifetime. The free energy cost of forming a liquid droplet is positive and increases with size here. This has been observed in a colloidal system by Savage and Dinsmore ${ }^{100}$. The liquid droplet has to form, and then persist for long enough for the crystal phase to nucleate inside it. The nucleation rate would then have the non-classical form

$$
\text { rate }=\nu_{l} \exp \left(-\Delta F_{c l}^{*} / k T\right) \exp \left(-\tau_{x} / \tau_{f l}\right)
$$

where $\nu_{l}$ is the characteristic frequency for particles entering and leaving the transient liquid droplet and $\Delta F_{c l}^{*}$ is the free energy of a liquid droplet that is large enough to accommodate a nucleus of the crystal. The final factor is the probability that a droplet that has become large enough to accommodate a crystal nucleus will persist long enough for the crystal phase to nucleate. This should depend exponentially on the timescale for the crystal phase to nucleate in the droplet, $\tau_{x}$, if this timescale is much larger than the characteristic timescale for fluctuations in size of the nucleus at the top of the barrier, $\tau_{f l}$.

Note that the rate decreases exponentially with the nucleation time of the crystal in the liquid droplet, $\tau_{x}$. This time is expected to increase exponentially with the barrier to nucleation of the 
Figure 11: Schematic showing three possible locations for nucleation in a system that has phase separated to form a dilute phase (the white area) containing a droplet of the concentrated phase (the pale blue droplet). The nucleus in each location is shown as a dark-red hexagon. Location 1): the nucleus forms in the dilute phase. Location 2): the nucleus forms in the concentrated phase. Location 3): the nucleus forms at the interface between the two phases. We have shown the nucleus at the interface forming in contact with an impurity particle (black square) also at the interface. Nuclei in locations 1) and 2) can also form in contact with impurities.

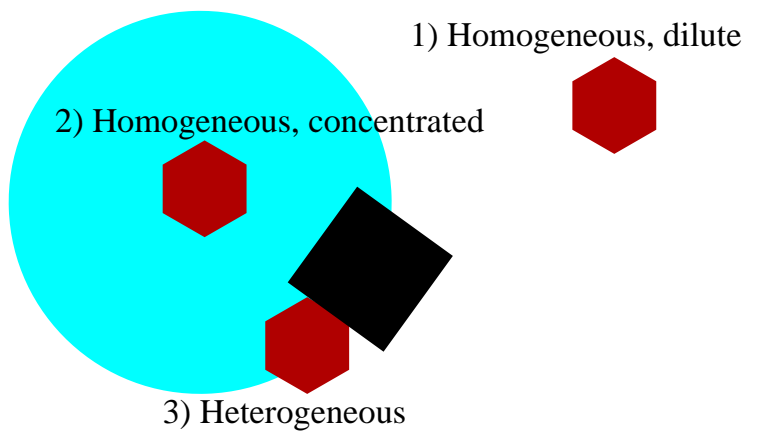

crystal phase in the droplet. Thus, the nucleation rate via a transient droplet is expected to be very slow unless the nucleation barrier to crystallisation in the droplet is low. If the barrier is not low the rate at which inherently transient liquid droplets form and then persist for the required very long time, is very low.

Finally, the above discussion ignores the role of solid impurity particles and surfaces in nucleation. But it should be noted that even outside vapour/liquid coexistence, microscopic amounts of liquid can appear in pits, slits, pores etc.. This is called capillary condensation. This is discussed in the section on nucleation in wedges and pits. Note that nanoscale structures in solution would easily be enough to allow capillary condensation to occur inside them in solution. This may be why such structures made from gelatin aid lysozyme crystallisation ${ }^{112}$.

\section{Lysozyme}

Vekilov and coworkers have studied solutions of the protein lysozyme. They find that they contain clusters of $10^{5}$ to $10^{6}$ molecules and that these large clusters affect crystallisation ${ }^{113-115}$. These clusters are present at low concentrations (1 ppm of the protein) and are outside the dilutesolution/concentrated-solution coexistence. It is very hard to see how these can form without impurities. In particular, charge effects, as studied by Hutchens and Wang ${ }^{64}$ cannot explain clusters of this size. The impurities could be irreversibly aggregated lysozyme molecules. Parmar et al. ${ }^{11}$ found evidence for non-equilibrium irreversibly formed aggregates of lysozyme, see Fig. 3. These affected crystallisation. Note that it is well known that liquid clusters can be stabilised by impurities ${ }^{12,13,62,63,116}$. The concentration of clusters is then controlled by the impurity concentration.

Thus it seems likely that in at least lysozyme and possibly all or most protein solutions, crystallisation is affected by the dynamic clusters of protein molecules, these may be stabilised by impurities, which may themselves be aggregated protein ${ }^{11}$, see Fig. 3 . Proteins are clearly different to smaller molecules, such as pharmaceuticals - which cannot unfold. However, even small molecules could cross-link, e.g., via oxidation, and it would only require a single cross-linked aggregate to stabilise a liquid cluster outside a liquid/liquid transition, which could then go on to crystallise. Thus it may be useful to apply the methodology of Parmar et al. ${ }^{11}$ to systems such as solutions of small molecules. 
Figure 12: A simulation snapshot from the work of Mendez-Villuendas and Bowles ${ }^{108,109}$. It shows a crystalline nucleus of 71 gold atoms growing in a liquid droplet of 456 gold atoms. The atoms of the nucleus are shown in dark gold while the atoms in liquid environments are shown as pale and translucent. Note that the nucleus forms at the surface of the gold droplet, i.e., at the liquid/vapour interface. Reprinted from Ref. 108 with permission. Copyright 2007 by the American Physical Society.



\section{Nucleation of a crystal phase at the vapour/liquid interface}

When vapour/liquid phase separation has occurred under conditions where the equilibrium state is a crystal, this crystal can potentially nucleate in one of three places: the liquid, the vapour, and the interface. This is shown schematically in Fig. 11. A crystalline nucleus of gold that has formed at the liquid/vapour interface of a liquid gold droplet, is shown in Fig. 12. At any of these three locations it could be occuring in contact with a small impurity particle. Note that impurity particles tend to concentrate at interfaces, due to the same physics as that behind Pickering emulsions ${ }^{117}$.

Applying classical nucleation theory to nucleation at each of the three possible places for nucleation we obtain three rates: $\nu_{l} \exp \left(-\Delta F_{l}^{*} / k T\right), \nu_{v} \exp \left(-\Delta F_{v}^{*} / k T\right)$ and $\nu_{i} \exp \left(-\Delta F_{i}^{*} / k T\right)$. Here $\Delta F_{\alpha}^{*}$ is the nucleation barrier, and $\nu_{\alpha}$ is the frequency with which molecules join the nucleus, where $\alpha=l, v$, and $i$ for the liquid, vapour and interface, respectively.

Now, the nucleation free-energy barrier comes from the free-energy cost of creating the interface around the nucleus. If the nucleus forms at the vapour/liquid interface then as it grows although there is a free energy penalty due to the cost of creating the nucleus/liquid and nucleus/vapour interfaces, the nucleus actually reduces the area of the vapour/liquid interface. This is is illustrated in Fig. 13. Therefore, there is a negative contribution to the nucleus free energy equal to $-\Delta a_{v l} \gamma$, where $-\Delta a_{v l}$ is the change in area of the vapour/liquid interface that occurs when the nucleus forms, and $\gamma$ is the vapour/liquid interfacial tension. Due to this term, unless the crystal surface melts the lowest nucleation barrier should be the one at the interface, $\Delta F_{i}^{*}$. If all phases are fluid (not glassy), the three $\nu_{x}$ should be comparable and nucleation should occur at the interface. This is the case for gold, see Fig. 12, where the nucleus is clearly forming at the interface ${ }^{108,109}$. If the crystal does surface melt then $\Delta F_{l}^{*}$ should be the lowest nucleation barrier. This is the case for the Lennard-Jones model, where the crystalline nucleus forms in the bulk of droplets, away from the interface $^{66,103}$.

However, in a number of cases, e.g., proteins and calcium carbonate, the molecular dynamics in the concentrated-solution phase are slow, i.e., the it is glassy. In these cases, we expect $\nu_{l} \ll \nu_{i} \ll \nu_{v}$, i.e., the dynamics at the interface is likely to be much faster than in the bulk concentrated solution but much slower than in the surrounding dilute solution. Clearly, if the molecular dynamics is 
Figure 13: Schematic showing two possible locations for nucleation at an interface, together with the interfacial areas that disappear when the nucleus forms. As in Fig. 11 the interface is between coexisting vapour and liquid phases shown in white and pale blue, and the solid particle at the interface is shown in black. The nuclei are dark red, and the interfaces that disappear when they form are yellow. The vapour/liquid interfaces that disappear when the nucleus forms are shown in pale yellow, while the particle/liquid and particle/vapour interfaces are shown in darker yellow.

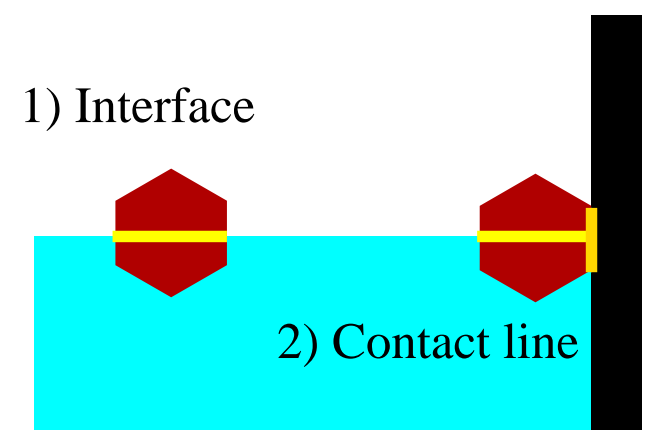

completely arrested in the concentrated-solution phase then nucleation cannot occur there, and so it will occur either on the surface of clusters of this glass, if this surface is still mobile, or in the vapour.

A final point is that due to the interface, the nucleation barrier there could easily be $10 k T$ or more lower than in the vapour, then nucleation will occur at the interface even for $\nu_{i} / \nu_{v} \ll 1$. However, if the bulk of the concentrated solution phase is arrested then growth will occur into the dilute solution phase, and crystals will start at the interface and grow out into the dilute solution.

\section{Nucleation at a contact line}

Nucleation commonly occurs on solid surfaces and solid particles will tend to be attracted to vapour/liquid interfaces, due to the physics responsible for Pickering emulsions ${ }^{117}$. Thus when nucleation is observed at a vapour/liquid (or dilute/concentrated solution) interface it may be occurring on the surface of a solid particle at that interface. This is illustrated in Fig. 11. When a vapour/liquid interface hits the surface of a solid particle, there is a contact line. This is also called a three-phase line as it is line along which three phases meet. Contact lines also arise when there is a droplet of liquid sitting on a solid surface.

At a contact line 3 interfaces meet and so when a nucleus forms there it replaces more interface than when it forms just at a vapour/liquid interface, where it can only replace one interface. This is illustrated in Fig. 13. So at the contact line the free-energy of the nucleus is lowered due to the disappearance of vapour/liquid, solid/liquid and solid/vapour interfaces, all of which can cost free energy. I have studied a simple lattice model (which does not show the analogue of surface melting) and indeed nucleation is fastest along the contact line ${ }^{118}$.

However, some crystals, e.g., ice, surface melt, i.e., the interfacial free energy cost of a direct crystal/vapour interface is larger than the sum of a crystal/liquid and a liquid/vapour interface. Then the crystal nucleus should avoid the vapour/liquid interface and hence the contact line. Nucleation is then expected on the solid surface but in the liquid; see the ice section for a discussion.

This picture of surface-melting crystals nucleating at the solid/liquid interface and non-surfacemelting crystals nucleating at the contact line may be too simple. Djikaev and Ruckenstein ${ }^{119}$ have considered the thermodynamics of nucleation at a three-phase line. In addition to the surface freeenergies there is a line tension ${ }^{31,120}$. This is the free energy associated with the contact line itself (in addition to the bulk and surface terms). It has dimensions of energy per unit length and can be positive or negative. If the line tension of the water/vapour/particle three-phase line is large and positive then this would reduce the nucleation barrier there. However the value of the line tension 


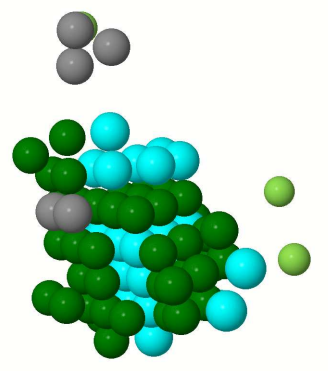

Figure 14: Snapshot of a configuration from a nucleation pathway. It is from the crystallisation of a liquid droplet of 850 Lennard-Jones molecules. Only the particles with local crystalline ordering are shown, these particles are in a liquid droplet but the particles in liquid-like environments are not shown. The cluster is studied at the reduced temperature $T^{*}=0.45$, as in the work of van Meel et al. ${ }^{66}$. The configuration shown is a quenched version of a configuration with a largest crystalline cluster of 200 crystalline molecules ${ }^{121}$. This is just over the barrier for crystallisation. Particles with fcc, hcp, decahedral and icosahedral local ordering are cyan, dark green, grey and light green, respectively. The local ordering of the particles is determined by the common-neighbour analysis of Honeycutt and Andersen ${ }^{122,123}$. Note that there is relatively large fcc domain (cyan) in the bottom right of the nucleus. This domain is limited by stacking faults at the top and left, these stacking faults show up as planes of molecules in locally hcp environments (dark green). There is also a five-fold axis forming along an edge of this fcc domain, note the two grey molecules in a locally five-fold symmetric environment and compare this snapshot with Fig. 15A), B) and D), which all have full formed five-fold axes. Simulation configuration courtesy of A. Page. This and Fig. 15 were produced using $\mathrm{Jmol}^{124}$.

is unknown.

Computer simulations will probably be required to understand freezing at a surface and along the three-phase line. But as ice surface melts there is no strong theoretical reason for expecting the free energy barrier for ice nucleation to be lowest at the contact line. As an example of systems which do not surface melt, we can consider gold. This does not surface melt ${ }^{108}$, and so a gold liquid droplet on a surface would be expected to freeze from the contact line.

\section{Defects}

Crystals are never perfect, the crystal lattice always has defects. The role of defects in crystal growth has been studied extensively. It is sufficiently standard to be discussed in textbooks ${ }^{125}$, and it has been appreciated since the work of Frank ${ }^{126}$ in the 1940s that screw dislocations can dominate the growth of crystals at low supersaturations. At low supersaturations growth at a screw dislocation is much faster than on the surface of a perfect defect-free crystal. This is because growth of an extra layer on the surface of a perfect crystal requires that this new layer nucleate. Nucleation is not required at a screw dislocation. At low supersaturations this nucleation barrier is large and so growth via layer-by-layer nucleation is extremely slow.

So, defects can qualitatively change the growth behaviour of crystals. Can they also qualitatively change the nucleation behaviour? There has been essentially no work on this, so we do not know the answer. However, given the vital role they play in growth at low supersaturations it seems likely that in at least some systems they are also important in nucleation.

To affect nucleation, the defects would need to form either before the top of the barrier is reached, or at the latest around the top of the barrier. Page ${ }^{121}$ has studied the formation of defects in the nuclei formed during the crystallisation of a cluster of 850 Lennard-Jones particles. She did find defects, but was only able to study nuclei that are little past the top of the nucleation barrier. The 
simulation snapshot in Fig. 14 is for a nucleus with 200 molecules identified as crystalline, whereas at the top of the barrier only around 160 molecules are identified as being crystalline. Note that there is clearly a defect forming in the nucleus, so defects can clearly form early on in crystallisation.

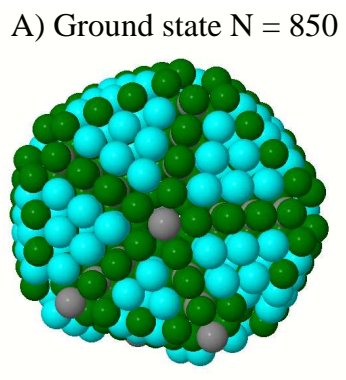

C) Stacking defects

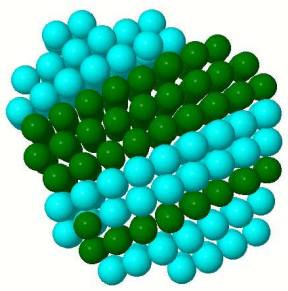

B) Ground state $\mathrm{N}=823$

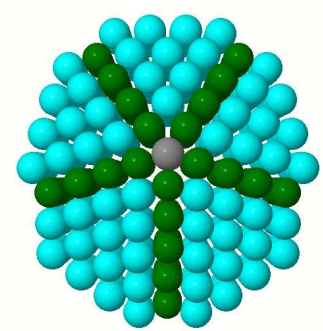

D) Partial decahedron

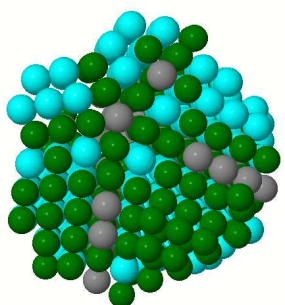

Figure 15: Figure showing defect patterns in crystalline clusters ${ }^{121}$. A) is the (icosahedral) ground state for $N=850$, and $\mathrm{B}$ ) is the (decahedral) ground state for $N=823$. Both configurations are from Xiang et al. ${ }^{127}$, see also the Cambridge Cluster Database ${ }^{128}$. C) and D) are obtained by crystallising liquid droplets of $N=850$ Lennard-Jones particles at $T^{*}=0.45^{121}$. Snapshots C) and D) courtesy of A. Page. Particles with fcc, hcp and icosahedral local ordering are cyan, dark green and grey, respectively. The disordered particles in the configurations of C) and D) are not shown. In $\mathrm{C}$ ) the crystalline ordering is such that all the close-packed planes are parallel but it is neither a single fcc nor a single hcp crystal as it is disrupted by stacking faults. D) is a partial decahedron with 3 relatively large fcc domains and 2 fragmentary ones, separated by 2 well-formed stacking faults (hcp layers) and 3 fragmentary ones. These stacking faults meet along an axis of five-fold symmetry. All 4 configurations have the local ordering of all particles determined by the common-neighbour analysis of Honeycutt and Andersen ${ }^{122,123}$. The configurations of C) and D) have been quenched from $T^{*}=0.45$ to $T=0$. The quenching is done by putting a configuration from a simulation of crystallisation at $T^{*}=0.45^{66,121}$, into a short Monte Carlo run at $T^{*}=0$. The short $T^{*}=0$ quenching run helps in identifying the locally fcc, hcp, etc. molecules with the common-neighbour analysis.

This work is not conclusive and the situation in other systems is completely unclear. Essentially all work completely neglects defects but we simply do not know whether neglecting defects is a moreor-less harmless approximation in most cases, or whether neglecting them results in large errors. We also do not know whether defects slow down or accelerate nucleation. It should be noted that the formation of defects themselves can involve nucleation ${ }^{2}$ and hence be slow, and that the standard techniques used in computer simulation can fail in the presence of a source of slow dynamics ${ }^{129-132}$. Thus existing simulation results may be missing defects due to problems with the algorithms.

As there are so few results in simulation and no experimental results that I know of, I will just briefly some preliminary results by Page ${ }^{121}$, and outline what is known about crystalline clusters and what consequences this may have for crystallisation. The critical nucleus is a small crystalline cluster. Such clusters have been extensively studied ${ }^{133-138}$ and it is now well established that the 


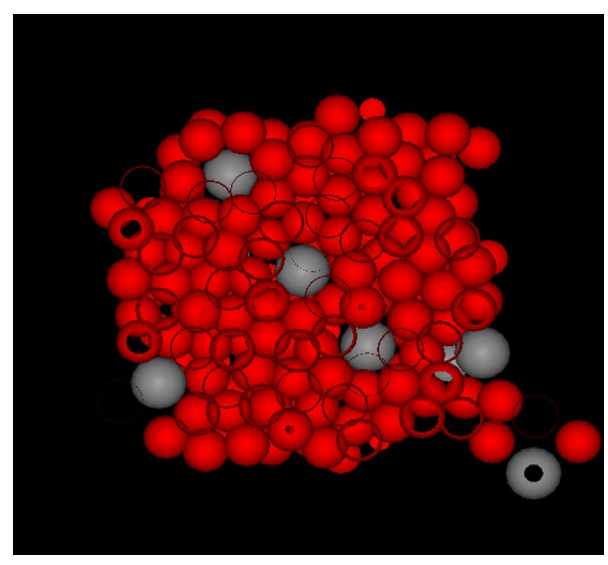

Figure 16: Figure from Anwar et al. ${ }^{139}$ showing a computer simulation configuration obtained on attempting to crystallise a system of Lennard-Jones molecules (shown as red spheres), in the presence of impurity molecules (grey spheres) ${ }^{139}$. The impurity molecules have a high affinity for the crystallising molecules and so are incorporated in the growing crystal, but are larger than the crystallising molecules and so strain the growing lattice. This strain can arrest crystal growth when the crystal is still microscopic. Reprinted from Ref. 139 with permission. Copyright Wiley-VCH Verlag GmbH \& Co. KGaA.

free energy is not minimised by the small crystallite being a defect-free piece of bulk lattice, it is minimised by an often intricate pattern of defects. Two example minima, chosen because of their different defect patterns, are shown in Fig. 15A) and B). They are energy minima, which are the freeenergy minima at zero temperature, and are for crystalline clusters of 850 and 823 Lennard-Jones molecules.

Defects are present at equilibrium in clusters, essentially because a chunk of a bulk lattice typically has a very high surface free energy. This is partly due it being far from spherical and partly due to it exposing faces with low coordination number. Introducing defect planes and lines can produce a more spherical crystallite with a lower surface free energy, and if this reduction in the surface free energy is large enough to overcome the cost of the defects, at equilibrium the cluster will have these defects.

The question then is: Given that defects can reduce the free energy, does the growing nucleus contain defects, or is it closer to a piece of the bulk crystal, with a correspondingly higher free energy? If the nucleus does contain defects, then how do they form? The formation of defects has been studied in bulk crystals, they typically form near the surface ${ }^{2}$. However, in growing nuclei we simply do not know the answers to these questions.

It seems likely that the formation of defects will typically be kinetically controlled, i.e., the defects that form will those can that can form easily, not those that minimise the free energy. This is supported by some work by Page ${ }^{121}$ who on crystallising clusters got crystallites with the defect patterns shown in Fig. 15C) and D), which are not the equilibrium defect pattern, shown in Fig. 15A).

Note that the equilibrium defect patterns are rather elaborate, see Fig. 15A) and B), and so are kinetically difficult to form. In other words if defects are taken into account the free-energy landscape of crystalline clusters is complex and rugged. Crystallisation will take place over this rugged landscape, and so classical nucleation theory's sixth assumption is highly unlikely to be correct. Predicting the pattern of defects that form will probably be challenging but it may well be that defects play a crucial role in the nucleation of some important crystals and so it is a problem that should be addressed.

Finally, note there has been some work on the nucleation of hard spheres, where defects clearly form ${ }^{140,141}$, but these defects may be forming after the nucleation barrier has been crossed. Also, 
defects can play a role in arresting the growth of small crystalline clusters, potentially producing a solution or vapour of many small crystallites that may be stable for long periods. Thus the clusters Gebauer et al. ${ }^{56}$ observed in calcium carbonate solutions could be crystallites whose growth arrested after incorporating impurities. Anwar et al. ${ }^{139}$ used computer simulation to study crystallisation in a Lennard-Jones fluid in which there were impurities. Some impurities they studied were such that they attracted the molecules strongly and so were incorporated into the growing crystallites but were the "wrong" size for the lattice and so created strain in the lattice. This strain arrested growth of the crystallites, see Fig. 16 for an arrested crystallite. This microscopic mechanism discovered by Anwar et al. may also underlie at least some of the effects of impurities on the crystallisation of glycine found by Poornachary et al. ${ }^{4}$.

\section{Heterogeneous Nucleation: Flat Surfaces}

Homogeneous nucleation of crystals is probably rather rare, usually impurities are involved in nucleation. Frequently these impurities have solid surfaces and the nucleus forms on this surface. As I discuss in my earlier review ${ }^{1}$, within classical nucleation theory, the nucleation barrier is almost always lower at a surface than in the bulk. The nucleation barrier comes from the surface terms in the nucleus free energy and at the solid surface there are pre-existing surfaces whose free-energy cost has already been paid. These pre-paid costs are subtracted from the nucleation barrier, resulting in faster nucleation at the surface. This reduction in the barrier at a surface is generic and applies to crystals. So it is a plausible basic explanation for the common observation of crystallisation starting at surfaces. However, the interaction between the growing nucleus and the surface may be complex, particularly if the surface is crystalline or non-planar, and this complexity may lead to effects not included within classical nucleation theory.

See my earlier review ${ }^{1}$ for details of the effect of surface geometry on the nucleation of fluid phases, which is well described by classical nucleation theory. Essentially, for fluids the more concave a surface is, the lower the nucleation barrier is, i.e., the nucleation barrier decreases monotonically as we increase the curvature of the surface towards the nucleus. However, even for fluids, nucleation in pits can be more complex, for example it can be a two step process ${ }^{142}$.

In this section and the next I will review work showing non-classical nucleation behaviour on solid surfaces. These solid surfaces will include completely smooth surfaces, crystalline surfaces and amorphous surfaces. In this section I will discuss flat surfaces, and in the next I will review non-planar surfaces, such as wedges and pits.

\section{Surface phase behaviour}

Nucleation at a surface will of course be affected by any process or change of state that occurs at that surface. Therefore, to understand nucleation of a bulk crystal at a surface, it is helpful to briefly consider surfaces themselves.

Surfaces exhibit a range of behaviour that can be richer than that found in the bulk. For introductions to this, see for example the classic review of de Gennes ${ }^{143}$ or the more recent review of Bonn and Ross ${ }^{144}$. These cover surface phase transitions, i.e., changes of state where the surface goes from one surface thermodynamic phase to another. An example is when, on cooling to near a liquid/vapour transition, a thick liquid film suddenly appears on a surface. This is called a prewetting transition and is a phase transition that occurs at the surface. This sudden appearance of a liquid film will cause a jump in the nucleation barrier of a bulk phase, such as a crystal, at the surface $^{145}$.

The surface layer of a liquid in contact with a solid surface can also freeze, i.e., acquire crystalline ordering, before the bulk freezes. This is illustrated in Fig. 8 For example, Castro et al. ${ }^{146}$ studied alkanes and alcohols on graphite and found evidence that layers of the alkanes and alcohols froze above the bulk melting point. Ocko et al. ${ }^{147}$ also studied alkanes, they found freezing of layers at the liquid/air interface. This is called pre-freezing and is expected to reduce the nucleation barrier to 
crystallisation in the bulk. Indeed, Page and Sear ${ }^{76}$ studied the effect of pre-freezing in the system of Lennard-Jones molecules at a smooth attractive wall. A crystalline layer that has formed via pre-freezing is shown in Fig. 8. In this system, once a crystalline layer has formed at the surface, the barrier to nucleation of the bulk crystal is effectively zero. Here there is no nucleation barrier to layer-by-layer growth of the crystal into the bulk.

Another example is the simple system of hard spheres near a hard wall ${ }^{148-150}$. For hard spheres at a hard wall pre-freezing seems to be first-order ${ }^{148}$. If pre-freezing is first order then there will be an associated nucleation barrier. However, as in the Lennard-Jones system, once one layer at the surface has ordered then there appears to be little or no barrier to the freezing of the 2nd, 3rd etc. layers ${ }^{76,148}$.

Thus in both hard spheres and Lennard-Jones fluids, we find that there may be only one barrier to the formation of a crystalline phase but this can be the nucleation barrier of a surface transition, not that for bulk crystallisation. This is the surface analogue of the situation found by Chen et $a l .{ }^{103}$ and by van Meel et al. ${ }^{66}$ where the rate limiting step for crystallisation was nucleation of an intermediate liquid phase.

Nucleation often occurs on microscopic impurity particles and then it seems unlikely that a prefreezing transition occurring on the impurity surface will be directly detected. However, hysteresis associated with surface transitions may show up as history-dependent nucleation rates for the bulk crystal.

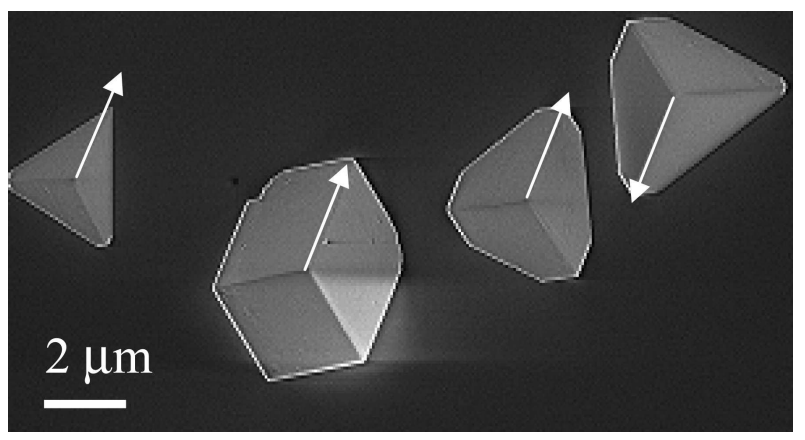

Figure 17: SEM images of calcite precipitated on mica, from Stephens et al. ${ }^{151}$. The crystals were grown from a $10 \mathrm{mM}$ solution for 1 hour. The arrows distinguish the two different orientations of the crystals. Note that the crystals are aligned, suggesting epitaxial nucleation and or growth. The crystals only aligned if the mica surface was "weathered" in an atmosphere of 35 to $40 \%$ humidity for approximately 1 hour, prior to crystallisation. Thus, the nucleation process may be complex. Reprinted from Ref. 151 with permission. Copyright 2010 American Chemical Society.

\section{Nucleation on crystalline surfaces}

When a crystal nucleates on a crystalline surface it is possible that the crystal lattice of the nucleus will form in registry with the crystal lattice of the surface. The nucleation is then epitaxial. Nucleation cannot be observed in experiments on molecular and ionic systems. However, it is sometimes observed that crystals have formed with their crystal lattices aligned with that of the surface on which they have grown. See Fig. 17 for an example. This behaviour is consistent with epitaxial nucleation and growth.

If the crystal lattices of the surface and nucleating phase are the same, e.g., both are bcc, the lattice constants are the same, and the molecules of the surface attract those of the nucleus, then nucleation is expected to be rapid. This would be the case for example, if it is not nucleation of a new phase but nucleation of a new layer on top of a growing crystal. However, for nucleation of one substance on the surface of a different substance, then in general at least the lattice constants will be different and the two lattices may have different symmetries as well. These differences are 
expected to increase the nucleation barrier.

Little work has been done on epitaxial nucleation. If the lattices of the nucleus and surface are the same with just differing lattice constants then it has been known for 50 years that the lattice mismatch $\Delta a$ causes strain in the nucleus ${ }^{152}$. This strain contributes a term to the free energy that scales as the nucleus volume times $\Delta a^{2}$. In turn, this will effectively reduce the supersaturation. In a simple model, the effect of this on the nucleus free energy is illustrated in Fig. 18A, compare the black (no strain) and red (strain) curves. Thus the nucleation rate varies as $\exp \left[-A /(\Delta \mu-B \Delta a)^{2}\right]$, where $A$ and $B$ are constants. This prediction for the effect of a lattice mismatch on nucleation has not been tested quantitatively, in either simulation or experiment.

Van Meel et al. ${ }^{153}$ studied the nucleation of an fcc crystal, in shallow pits in a fcc crystalline solid surface. They used computer simulations of a simple model. When the match between the lattice constants of the surface and nucleus was almost perfect, nucleation was rapid. However, van Meel et al. found that even a $4 \%$ mismatch in the lattice constant dramatically affected epitaxial nucleation, a nucleus formed but it was split by a defect. This can be seen in Fig. 18B where there is a clear crack in the nucleus. The crack is presumably caused by the crystal nucleus cracking under the strain of conforming to the too-small lattice constant of the surface. On increasing the lattice mismatch to $10 \%$ they found that nucleation stopped altogether. Note that a type of defect called a dislocation can relieve strain ${ }^{2,152}$. However, dislocations were not observed. A dislocation has a free energy cost, which may be high, and the dislocation itself will need to nucleate in the nucleus.

\section{Heterogeneous Nucleation: Wedges and Pits}

Classical nucleation theory predicts that the free energy barrier to nucleation is generically lower at surfaces than in the bulk, and generically lower at concave surfaces than at either flat surfaces or convex surfaces. Thus, we expect that the nucleus will form at concave parts of the surface in contact with the parent phase. The reason for this is simple geometry: the more the solid surface curves towards the nucleating phase, the larger is the area of the surface of the nucleus that is in contact with the solid surface. This then reduces the area of the interface between the nucleus and the phase it is nucleating in, and as it is the free-energy cost of this interface that is the source of the nucleation barrier, the barrier is then lower.

\section{Wedges}

The first non-planar geometry we will consider is the wedge. This is formed from 2 smooth flat surfaces that meet along a line at some internal angle $\beta$, see Fig. 19A and 20c. As the angle $\beta$ decreases and the wedge becomes narrower, the nucleation rate monotonically and rapidly increases.

This behaviour is well described by classical nucleation theory ${ }^{121}$. The classical nucleation theory prediction for the nucleation barrier in a wedge is that the barrier has the form $\Delta F^{*}(\theta, \beta)^{154}$. Here $\theta$ is the contact angle the interface between the nucleus and the bulk phase makes with a flat surface. For $\beta=180^{\circ}$ the wedge reduces to a plane and the nucleation barrier disappears at wetting, when $\theta=0^{\circ}$. For a wedge, $\beta<180^{\circ}$ and the nucleation barrier at constant $\theta$ decreases monotonically as $\beta$ decreases. The barrier becomes zero when $\beta=180^{\circ}-2 \theta$. Thus in a wedge we can have barrier-less nucleation of a fluid phase even when this fluid has a significant contact angle on the solid surface and so is far from wetting it. The disappearance of the nucleation barrier is associated with a surface phase transition called filling ${ }^{155,156}$.

This is all for fluids in a wedge. Page and Sear ${ }^{123}$ showed that the nucleation of crystals in a wedge is much more complex. They studied the nucleation of the crystal phase of the Lennard-Jones model from the liquid phase, in wedges with smooth walls. The Lennard-Jones model has two crystal polymorphs: fcc and hcp, which are essentially equally stable ${ }^{157}$. If we consider the fcc polymorph, the densest crystal planes are the $\{111\}$ planes. Thus if the surfaces of a wedge are smooth and attract the particles, it is favourable to have $\{111\}$ planes directly in contact with both surfaces of 
(A)
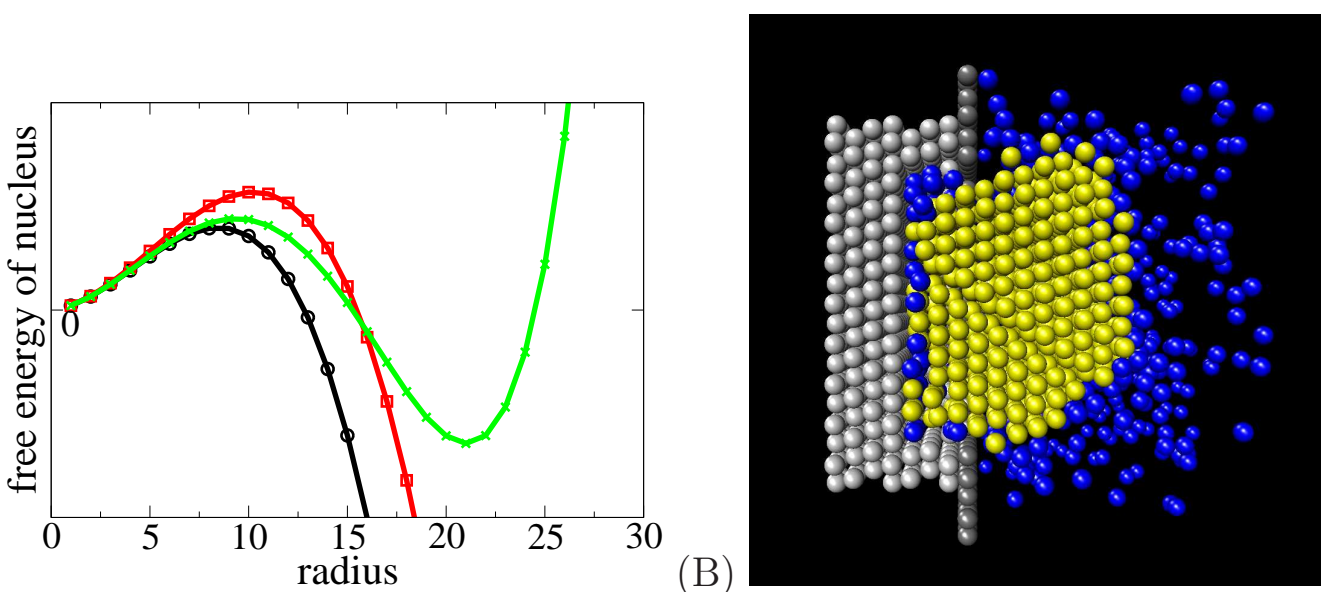

Figure 18: (A) Plot of the free energy of (cubic) nuclei, as a function of their size. The plot includes free energies for an unstrained nucleus (black curve), a nucleus strained due to a lattice mismatch (red curve), and a nucleus strained due to conforming to a curved surface (green curve). The free energies are obtained from a simple lattice model of strain combined with classical nucleation theory. (B) A simulation snapshot of a nucleus that has formed in contact with a shallow cylindrical pit cut from an fcc crystal. This substrate is oriented so that it has $\{111\}$ planes facing the fluid. The cylinder has a radius $R=7$ and depth $D=3$. The snapshot is a cross-section through the centre of the pit. The lattice constant of the substrate is $\approx 4 \%$ smaller than that of the nucleating crystal. This mismatch has caused a defect to form which has cracked the nucleus. It has also arrested the growth of the nucleus. The dark-grey, light-grey, blue and yellow particles, are purely repulsive wall particles, wall particles that attract the fluid particles, liquid particles and crystalline particles, respectively. By liquid and crystalline particles we mean particles in locally liquid-like and crystalline environments. (B) is adapted from Ref. 153 with permission. Copyright 2010 by the American Physical Society.

the wedge. Then dense planes of particles are in contact with both surfaces and so are in the energy minima at these surfaces.

The problem with having $\{111\}$ planes along both surfaces of a wedge is that in a defect-free unstrained fcc lattice, pairs of $\{111\}$ planes can only have certain angles between them: these are $0^{\circ}$, $70.5^{\circ}, 109.5^{\circ}, \ldots$. The $0^{\circ}$ angle is relevant to nucleation in slits with parallel walls, although here for a crystal to form without strain the distance between the walls must equal the width of an integer number of lattice planes ${ }^{158,159}$.

So, in an fcc crystal there are pairs of $\{111\}$ planes at an angle of $70.5^{\circ}$ to each other, and therefore a nucleus of an fcc crystal fits perfectly into a wedge with an internal angle of $\beta=70.5^{\circ}$. Page and Sear ${ }^{123}$ found that nucleation was rapid in wedges with this value of $\beta$. They also found that the nucleation rate was a local maximum at $\beta=70.5^{\circ}$. At both smaller and larger values of $\beta$ the fcc crystal cannot fit perfectly into the wedge and so the nucleation rate is lower. This is qualitatively different from the prediction of classical nucleation theory, which predicts that the nucleation rate increases monotonically as $\beta$ decreases. Also, analysis of the crystal shows that it is a single fcc domain without stacking faults. See Fig. 19A for a crystal that has formed in a $\beta=70.5^{\circ}$ wedge. This is different from homogeneous nucleation, where defects form, see Fig. 15C and D.

Page and Sear ${ }^{123}$ also found that in narrow wedges crystals nucleate slowly as the crystal cannot fit into the narrow point of the wedge, see Fig. 19B for a crystal that has formed in a wedge with 


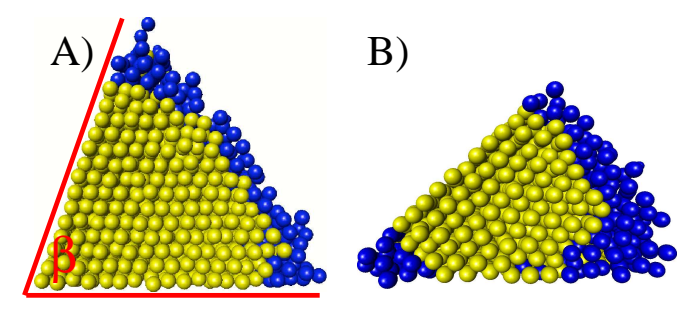

Figure 19: Simulation snapshots of Lennard-Jones crystals in wedges, from Page and Sear ${ }^{123}$. Both A) and B) are cross-sections of the final crystal found after the liquid droplet has completely crystallised. We are looking along the axis of the wedge, and the point of the wedge is at the bottom left. The surfaces of the wedge are shown schematically as red lines in (A) only. (A) and (B) are snapshots of crystals in wedges of angles $\beta=70.5^{\circ}$, and $45^{\circ}$, respectively. The molecules in a locally crystalline environment are shown in yellow while those in a fluid environment are shown in dark blue. The temperature $T^{*}=0.55$. Adapted from Ref. 123 with permission. Copyright 2009 American Chemical Society.

angle $\beta=45^{\circ}$. Note that right at the point of the wedge there is a small pocket of still disordered particles (shown in blue). The nucleation rate for a crystalline phase in a $45^{\circ}$ wedge is much lower than in a $70^{\circ}$ wedge whereas the nucleation rate for a new fluid phase would be much higher in the narrower wedge.

Well before these simulations were performed, Ward and coworkers ${ }^{160-162}$ crystallised molecular crystals in wedges (with obtuse angles $\beta$ ) made by cleaving a crystal. They cleaved crystals to make wedges, and then observed preferential nucleation of benzoic acid and other molecules along the line of the wedge ${ }^{160-162}$. The crystals formed at a fixed angle to the wedge and to the lattice of the substrate. For example, Mitchell et al. ${ }^{160}$ looked at the crystallisation of ROY on cleaved surfaces of pimelic acid. They found that the crystals that formed aligned with the lattice of the surface, and also that one surface of pimelic acid strongly favoured one of ROY's polymorphs. It favoured the "yellow needle" polymorph. This is shown in Fig. 20. The crystals are shown in Fig. 20a while $20 \mathrm{c}$ is a schematic showing how the ROY crystal aligns with the lattice of the crystal it has formed on.

Note that the simulation results were for smooth walls whereas these experiments were for wedges with crystalline surfaces, so the nucleation in experiment may well be epitaxial. However, experiment and simulation agree that wedge-type geometries can potentially induce nucleation, control crystal alignment, and favour one polymorph over others. All these are useful properties.

\section{Curved surfaces}

The wedges and ledges we have considered are made of flat planes but surfaces can also be curved. Curvature is expected to inhibit nucleation of a crystalline phase. This is different from the classical prediction and from the nucleation of a fluid. For a fluid, curving towards the nucleus reduces the barrier while curving away increases it. However, crystals have a lattice and if this lattice has to bend to conform to a curved surface then there is a free-energy cost due to lattice strain. Of course this affect is completely absent in fluids.

At small curvatures (with respect to the lattice constant of the nucleus), the lattice strain is as follows. If a surface has some small curvature, i.e., a large radius of curvature $R_{c}$, and the nucleus conforms to this surface then the strain free energy scales as $E R^{5} / R_{c}^{2}$, where $E$ is the elastic modulus of the crystal, and $R$ is the radius of the nucleus. Note that this is symmetric with respect to positive and negative curvatures, as it depends on the square of $R_{c}$. Thus for small curvatures, concave and convex surfaces are equivalent. They both strain the nucleus and so inhibit nucleation.

Elastic moduli are typically large, i.e., $\gg k T / v_{u c}$ with $v_{u c}$ the volume of the unit cell, and so for a critical nucleus that is approximately 10 molecules across then any curvature $R_{c}^{-1}$ more than 

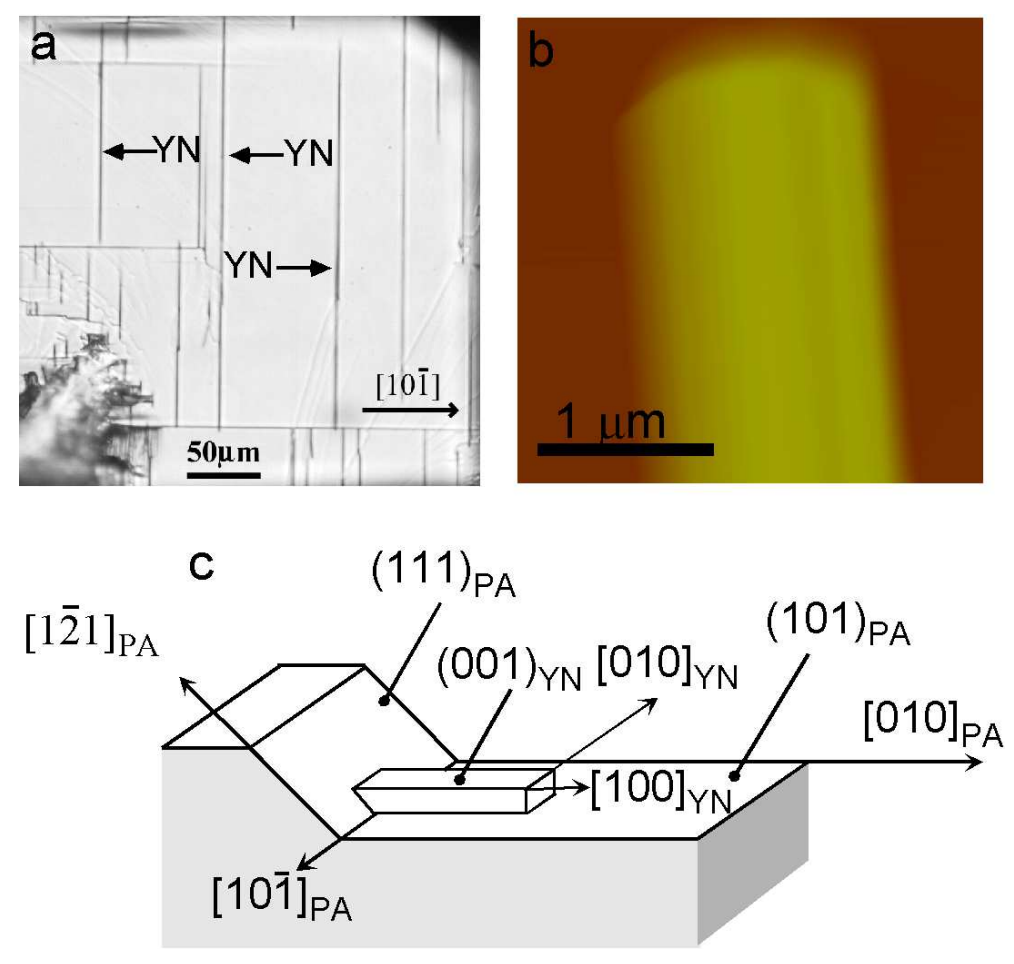

Figure 20: Crystallisation of the "yellow needle" (YN) polymorph of ROY on the surface of a cleaved crystal of pimelic acid, by Mitchell et al. ${ }^{160}$. The pimelic acid is cleaved to expose its (101) lattice plane. (a) is a photomicrograph of highly oriented YN crystals grown by sublimation on the cleaved (101) surface of a single crystal of pimelic acid. Some of the YN crystals, all of which are oriented vertically in the image and so perpendicular to the $[10 \overline{1}]$ direction of the pimelic acid lattice, are indicated by the arrows. The crystals are the dark lines, as the polymorph name suggests the crystals are needle like. (b) is an AFM image of a YN crystal on the (101) pimelic acid surface. (c) is a schematic representation of the YN crystal orientation, as determined by AFM goniometry. The YN crystals grow with their [100] direction perpendicular to the $[10 \overline{1}]$ ledge of the pimelic acid lattice and with their (001) face contacting the (101) plane of pimelic acid. Reprinted from Ref. 160 with permission. Copyright 2001 American Chemical Society.

approximately $10 \%$ of the inverse of the molecular diameter should prevent a nucleus forming. Cacciuto et al. ${ }^{163}$ studied the nucleation of crystals of hard spheres on the surface of larger hard spheres. They found rapid nucleation when the larger hard sphere was at least six times larger than the spheres of the crystal nucleus, However, if the larger sphere was smaller than that, nucleation did not occur in contact with this sphere's highly curved surfaces.

Interestingly, Cacciuto et al. ${ }^{163}$ also observed that as they grew, nuclei detached from the curved surface where they nucleated. This can be understood if we realise that the strain free energy increases as $R^{5}$, which is much faster than the increase of the free-energy gain due to being at the surface, which only increases as $R^{2}$. Thus small nuclei can form by bending to conform to a curved surface but as the nucleus grows then the strain energy will overwhelm the $R^{2}$ surface term that favours adhesion. Then it is favourable for the large nucleus to detach from the surface and relax the strain - which is what the nuclei of Cacciuto et al. appear to do. They simulated a specific simple system but the effect they observe should be generic. Also, note that the $R^{5}$-dependent strain free energy can lead to a minimum value in the free energy of the nucleus, see the green curve in Fig. 18A. Potentially, this could trap nuclei at a small finite size. 


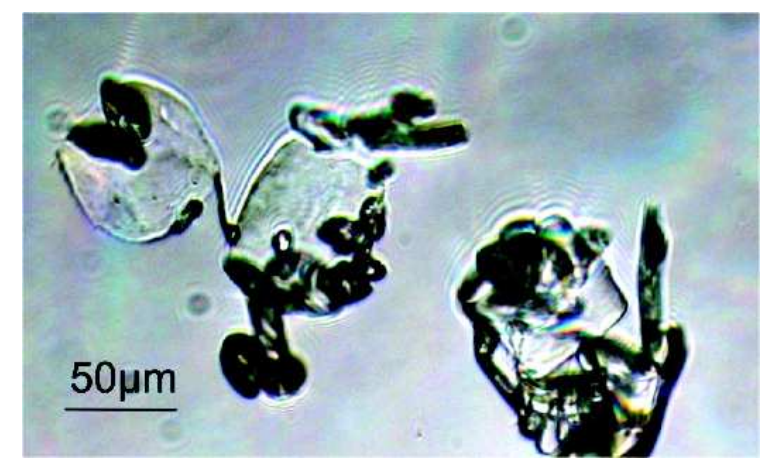

Figure 21: Aspirin crystals (dark) that have formed on microgel particles (translucent). The microgel particles are around $30 \mu \mathrm{m}$ across, and are made of cross-linked polyethylene glycol diacrylate (PEGDA) polymer. The particles are added to a supersaturated solution of $38 \mathrm{mg} / \mathrm{mL}$ aspirin solution in $38 / 62(\mathrm{v} / \mathrm{v})$ ethanol/water at $15^{\circ} \mathrm{C}$. The solution is stirred at $700 \mathrm{rpm}$. There are 15 $\mu \mathrm{g} / \mathrm{mL}$ of the microgel particles in the solution. Reprinted from Ref. 164 with permission. Copyright 2011 American Chemical Society.

\section{Pores and pits}

Surfaces may be rough, they can contain pits, pores, slits etc.. Here, by pits we mean an indentation in the surface that is not very far from circular and has a closed end. Pits are also sometimes called cavities. A pore is similar but does not have a closed end, it runs through the material. Some substances, known as porous media, are not solid at all but have pores running all through them. Examples are porous glass and gels. Slits are formed between two flat or approximately flat surfaces with a constant separation. In other words, pits and pores confine a volume of space in two dimensions, while a slit confines in one dimension.

Chayen et al. ${ }^{165}$ found that porous media is effective at inducing the nucleation of protein crystals. This was with a type of porous silicate with pores of range of shapes and sizes in the range 2 to $\sim 10 \mathrm{~nm}$. Zeolites ${ }^{166}$ and a porous medium made from carbon nanotubes and gelatin ${ }^{112}$ have also been used. Note that except for the zeolite, all these materials had pores a few protein diameters across and so possibly large enough to accommodate a nucleus of the crystal phase, but small enough to confine and strongly interact with this nucleus. Much earlier work by Turnbull ${ }^{167}$ considered the role of pits in both enhancing nucleation in metals, and as an explanation for historydependent nucleation. The use of porous or pitted surfaces is not only applicable to protein crystals, for example Diao et al. ${ }^{164}$ used gel particles to induce the nucleation of aspirin, see Fig. 21.

Inspired by the work on protein crystallisation, van Meel et al. ${ }^{153}$ used computer simulation to study nucleation in pits. The pits were in a model of an amorphous material and so had rough walls. They found rapid nucleation in the pits, see Fig. 22 for a snapshot of a crystal nucleus growing in a pit. The nucleus is growing inside a droplet of liquid that has formed in the pit via capillary condensation. The bulk was still in the vapour phase. The assumption is that the crystal/liquid interfacial tension is lower than the crystal/vapour interfacial tension, and therefore the free energy barrier for the nucleus in the pore is much lower than in the bulk. The system studied by van Meel et al. is near to, but not within a vapour/liquid transition.

Protein solutions often exhibit a dilute-solution/concentrated-solution transition, the analog of a vapour/liquid transition. Near this transition but when the bulk is still in the dilute-solution phase, pits in an attractive surface could fill with the concentrated-solution phase. This is also capillary condensation, and it would also cause the nucleation barrier to a crystal phase to decrease dramatically. As the nucleus forms away from the sides of the pit, no epitaxial match is required between the crystal and the solid. Indeed the solid can be amorphous as it is in the work of van Meel et al. ${ }^{153}$.

Thus this mechanism is not specific to protein solutions, it is only specific to solutions with 


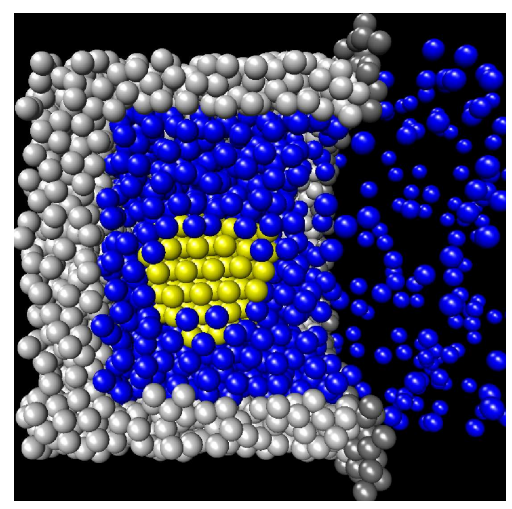

Figure 22: A simulation snapshot of crystallization from the work of van Meel et al. ${ }^{153}$. The snapshot is a cross-section through a simulation box. It shows a crystal nucleus (yellow) forming in a droplet of liquid (blue) in a pit with rough walls (silver). Note the layer of liquid particles between the yellow growing crystal and the walls. The surface is made of fixed particles that are equal in size to the model protein particles. All model protein and surface particles interact with the spherically symmetric potential with a short-range attraction of ten Wolde and Frenkel ${ }^{168}$. Fixed particles that attract the model protein particles are light grey; fixed particles that do not attract the model protein particles are dark grey. The model protein particles themselves are shown in blue and yellow. Blue particles are in a locally liquid-like environment, while yellow particles are in a locally crystalline environment. Reprinted from Ref. 153 with permission. Copyright 2010 by the American Physical Society.

dilute-solution/concentrated-solution transitions. Also note that the system only needs to be near the transition, it can still be in the dilute solution part of the phase diagram and so no bulk concentrated solution will form. Thus in experiment the role the concentrated-solution phase is playing in nucleation in pits may be hidden, although it should show up as a rapid increase ${ }^{110}$ in nucleation rate as a dilute-solution/concentrated-solution transition is approached. Galkin and Vekilov ${ }^{93}$ observed just such a rapid increase in solutions of the protein lysozyme. The simulation results of van Meel et al. may be relevant to these experiments. Also, snow frequently forms in the Earth's atmosphere under conditions where metastable liquid water can form ${ }^{12}$, so ice nuclei may be forming in water-filled pits of hydrophilic aerosol particles.

Diao et al. ${ }^{169}$ have studied the nucleation of aspirin crystals from solution, on surfaces with and without pores, see Fig. 23. They studied nucleation on the surfaces of polymer films. To analyse their data they started from the observation that if one nucleation event is all that is required to induce crystallisation, then the probability $P(t)$ that nucleation has not occurred in a sample is $P(t)=\exp (-r t)$, where $r$ is the nucleation rate. To study this function many samples must be studied in order to obtain statistics. Each experiment in Fig. 23 was of 48 independent samples.

Then, if there are $N_{S A M}$ samples and $N_{N X}(t)$ have not crystallised at time $t$, then the fraction $f(t)=N_{N X}(t) / N_{S A M}$ is a good approximation to $P(t)$, for large $N_{S A M}$. Diao et al.'s ${ }^{169}$ results for this approximation to $P(t)$ are shown in Fig. 23. The naive expectation is then that this fraction $f(t)$ should be exponential. This will be the case if the nucleation is either homogeneous or occurs on surfaces that are similar in all 48 samples.

However, if the nucleation is heterogeneous and the surfaces present in each sample are different then the nucleation rate will be different in each trial and so the fraction of trials that have crystallised will not be an exponential function of time. Diao et al. ${ }^{169}$ found that they could fit some of their data with a sum of two exponentials. This suggests that for these experiments some samples had one intrinsic nucleation rate while the rest had a different rate. They then determined that the faster rate was associated with samples with porous surfaces while the slower rate was associated with polymer samples where, during synthesis, pores had not formed. Earlier work by Kabath et 


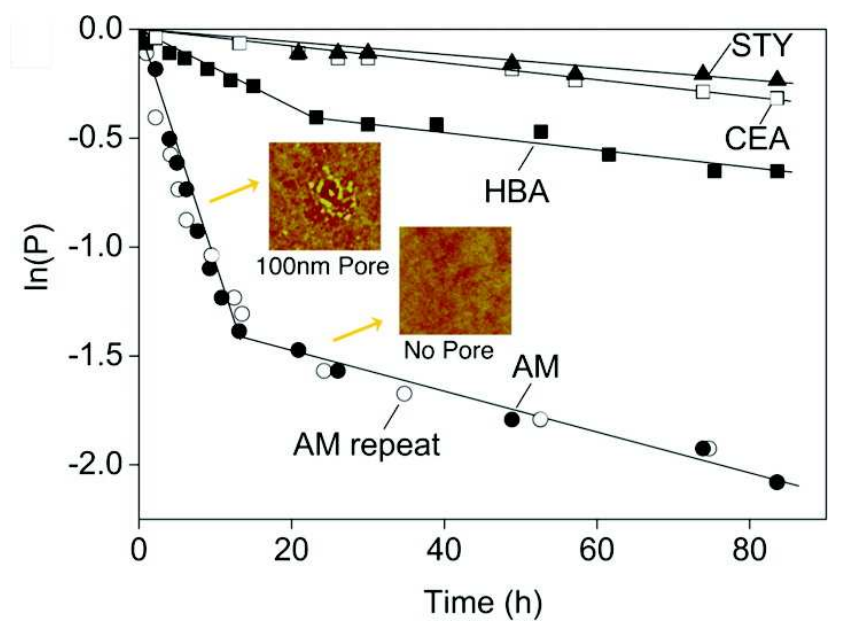

Figure 23: A plot of the probability $P$ that nucleation has not occurred in a sample, as a function of time in hours. This is the work of Diao et al. ${ }^{169}$, and is for the nucleation of aspirin from a supersaturated solution with a solvent that is a mixture of water and ethanol. The probability of crystallisation is obtained from the fraction of 48 independent samples (vials of solution) where crystallisation has occurred. In each vial there was a polymer film that is being tested for the efficiency with which it induces nucleation. STY, CEA, HBA and AM refer to films of different polymers, i.e., the polymer surface chemistry is being varied. See Ref. 169 for details of the polymers. The filled black and open symbols are independent repeats of the same experiment. Note that for AM and HBA the plots appear to be a straight line of one slope followed by a straight line of a different slope. This suggests two nucleation rates, with a faster rate (steeper slope) from substrates with $\sim 100 \mathrm{~nm}$ pores, plus a slower rate for the substrates that do not have pores. Reprinted from Ref. 169 with permission. Copyright 2011 American Chemical Society.

$a l .{ }^{170}$ on the nucleation of ice, also found two slopes and hence possibly two rates in a plot of the same type.

Thus, here pores appear to accelerate the nucleation of aspirin crystals; as with proteins ${ }^{112,165}$ rough surfaces are effective at inducing nucleation. The method used by Diao et al. is interesting. Data sets such as the HBA and AM curves in Fig. 23 which show a $P(t)$ that is not a simple exponential decay, immediately rule out homogeneous nucleation, as if nucleation is homogeneous the rate must be the same in every sample. Also, deviations from a single exponential directly provide information on how heterogeneous are the surfaces on which nucleation is occurring. The farther the curve is from a simple exponential the larger the spread of rates in the samples. Thus, plots of the type of Fig. 23 may be prove widely useful in studies of nucleation. Counting the number of crystals in a number of samples and comparing the result to a Poisson distribution, as Galkin and Vekilov $^{171}$, and Selimović et al. ${ }^{172}$ do, is also useful as a way of testing whether or not nucleation is occuring on heterogeneous surfaces and so is occuring at a range of nucleation rates.

\section{Laser-induced nucleation}

One of the most intriguing nucleation phenomena of the last 20 years is that of nucleation induced by high-power laser beams. A high-power, pulsed, laser is fired through a supersaturated solution and then crystallisation is observed. A control solution is not exposed to the laser beam and no nucleation is observed there. This was first observed by Garetz, Myerson and coworkers in 1996, in aqueous solutions of urea ${ }^{173}$. They used a laser in the near infrared, $\lambda=1.06 \mu \mathrm{m}$, where urea does not absorb, so they believe that nucleation was not induced by a photochemical process. They dubbed this process Non-Photochemical Laser-Induced Nucleation (NPLIN) ${ }^{173-176}$, and studied glycine in solution ${ }^{175,176}$ as well as urea. It should be noted that prior to the experiments with the laser, 
Garetz, Myerson and coworkers "age" their samples for several days. This is done by holding them at the final concentration of the trial, but at higher temperatures so they are undersaturated. It is unclear why this has an effect (although Fig. 3(B) shows an example of an "aging" process that affects nucleation). Alexander and Camp ${ }^{177}$ found that a similar laser beam could induce the crystallisation of potassium chloride, also from aqueous solution. Also, glycine has three polymorphs, and Zaccaro et al. ${ }^{175}$ and Sun et al. ${ }^{176}$ showed the laser-induced nucleation could change the polymorph produced. As polymorph control is often very important, this is a potentially very useful finding.

Let us consider the experiments on glycine solutions by Zaccaro et al. ${ }^{175}$, as a typical example of this work. The peak laser power was approximately $10^{13} \mathrm{~W} / \mathrm{m}^{2}$ which produces electric fields of $10^{8} \mathrm{~V} / \mathrm{m}$. The laser was pulsed with each pulse only $9 \mathrm{~ns}$ long; there were 10 pulses per second. The wavelength was $1.06 \mu \mathrm{m}$ giving a frequency of $10^{14} \mathrm{~Hz}$. At this frequency the glycine molecules cannot rotate to allow their permanent dipoles to follow the electric field. However, the electric field $E$ will induce dipole moments $\mu_{I N D}=\alpha E$, for $\alpha$ the polarisability of glycine. These induced moments in the glycine molecules will contribute to the glycine-glycine interaction. A strong induced attraction between glycine molecules will in effect further supercool the glycine solution in the laser beam, so reducing the nucleation barrier. But only for the 9 ns duration of the pulse.

Now, the polarisability of glycine is approximately $\alpha=10^{-39} \mathrm{C} \mathrm{m}^{2} / \mathrm{V}^{175}$. Polarisability is a tensor, this is an approximation to typical values of its components. The energy of interaction between two induced dipoles $r$ apart is

$$
u_{I N D} \simeq \frac{\alpha^{2} E^{2}}{4 \pi \epsilon_{0} r^{3}}
$$

Putting in the numbers for Zaccaro et al.'s experiments we obtain an energy of interaction of two urea molecules $1 \mathrm{~nm}$ apart of $10^{-25} \mathrm{~J} \simeq 10^{-4} k T$ at room temperature. As they appreciated, this is very small. See also the lattice-model simulations of Knott et al. ${ }^{178}$ who also find this result.

Even with a nucleus of 100 urea molecules, and so $100^{2}$ pair interactions, we only have a total energy change of $k T$ due to the laser E field. Also, the laser pulses are only $9 \mathrm{~ns}$ long, and this is not long enough for a nucleus to self-assemble. The diffusion constant of glycine in water is $\simeq 10^{-9} \mathrm{~m}^{2} \mathrm{~s}^{-1179}$ and so it takes a glycine molecule a fraction of a nanosecond to diffuse its own diameter. Ten nanoseconds is then not enough for a nucleus of 100 or more glycine molecules to form and grow over the nucleation barrier, before the pulse ends.

So it is clear that the observed nucleation behaviour cannot be explained by the effect of a light induced attraction on homogeneous nucleation. However, the nucleation is unlikely to be homogeneous, the nucleation is probably occurring in contact with small impurity particles in the solution. Now if such a particle is highly polarisable, e.g., if it is metallic, then due to this larger polarisability and its much larger size, the induced interaction energies between the nanoparticle impurity and a glycine molecule can easily be greater than $k T$. However, this still leaves the problem that this induced interaction only lasts 9 ns. We do not have good models of how molecules in a solution around a nanoparticle react to suddenly feeling a strong but very short lived attraction towards it. Future theoretical or simulation work could study this, and future experimental work could consider solutions doped with very low concentrations of metallic nanoparticles. Gold nanoparticles have been used to induce nucleation in protein solutions ${ }^{180}$, this was without a laser.

Hiroshi et al. ${ }^{181}$ also induced the crystallisation of urea from solution using a laser. However, they used a femtosecond laser intense enough to induce observable cavitation. Similar pulses have been used to induce the nucleation of other molecules, including the protein lysozyme ${ }^{182}$. Although we do not have a good model for this, the dramatic changes in temperature and pressure that cavitation causes will certainly strongly effect the nucleation rate and so it is probably unsurprisingly that nucleation can be induced. Modelling work needs to done to understand this phenomena, and also possibly to see if the weaker, longer nanosecond laser pulses used by Garetz, Myerson and coworkers, and by others, may be strong enough to induce very localised cavitation phenomena near a suitable impurity. Only a very small cavity could be enough to induce nucleation. 


\section{Conclusion}

We want to understand and control nucleation. Unfortunately, in experiments on molecular and ionic systems we cannot observe nucleation directly and it appears that it is very often occurring in contact with an uncharacterised impurity. For these reasons nucleation is a difficult problem, and often little attempt is made to understand it beyond the simple classical nucleation theory. This simple theory is clearly not adequate for many substances and may well be inadequate for the crystallisation of most substances.

However, the experimental work on ice formation ${ }^{13}$ shows that it is possible to quantitatively characterise the conditions where nucleation does and does not occur, and to get a fair understanding of what impurities are most effective at inducing nucleation. The same techniques should be able to provide a similar understanding in other molecular and ionic systems. Also, Muschol and coworkers ${ }^{11}$ have shown how using scattering to characterise impurities, and filtering to remove them, can reveal the role of impurities in nucleation. Diao et al. ${ }^{164,169}$ have shown how plotting the fraction of a set of samples that have crystallised shows up variations in nucleation rate from one sample to another, and Galkin and Vekilov ${ }^{171,172}$ have pioneered the counting of crystals in a number of samples to provide information about the nucleation kinetics. All these experimental methods are generally applicable and can be used to gain valuable data on the, typically poorly, characterised impurities nucleation is often occurring on.

To understand experiments, we rely on theory and simulation. Theory tells us that interfaces are key to nucleation, for two reasons. The first is that the free-energy barrier to nucleation comes from the interface around the nucleus, and the second is that nucleation is expected to very often occur at an pre-existing interface. Interfacial phenomena are now well understood, we have good models for wetting, capillary condensation, Pickering emulsions, etc. This understanding can now be very usefully applied to increasing our understanding of nucleation. Simulation will continue to provide most of our insights into the microscopic dynamics of nucleation. A significant amount of recent simulation work has moved on from studying homogeneous nucleation to studying heterogeneous nucleation. As heterogeneous nucleation is far more common than homogeneous nucleation, this is bringing experiment and simulation closer together. For all these reasons I believe the future is bright for both experimental and simulation research on nucleation.

In the introduction I characterised the conventional or classical theory of the nucleation of crystals as making six assumptions. In this conclusion it is perhaps useful to finish by going through these six assumptions, in each case considering examples of systems where this assumption fails. The list is:

1. Nucleation is a one-step process. It has been known for more than hundred years that crystals can form via multiple successive nucleation events which form a series of phases of increasing stability. For example, small molecules ${ }^{96}$, proteins ${ }^{11,92-95}$ and ice ${ }^{13}$, can all crystallise from a dilute solution (vapour) via a concentrated solution (liquid) phase. Also, many molecules can form one polymorph via nucleation on a less stable polymorph that forms first ${ }^{48,79,82-85}$.

2. The nucleus grows one monomer at time. The nucleus can only be studied in microscopic detail in simulation and so this assumption can currently only be tested in simulation. All simulations so far have shown nuclei growing more-or-less one molecule at a time. However, experiments on a number of systems have found evidence for clustering in supersaturated solutions. It may be that the nucleus here can grow by coalescence of these clusters, and so grow by tens or more molecules in a single step. Therefore, the validity of this assumption is under doubt in some systems. Either experiments which study the dynamics of these clusters, or simulations, are required to test this assumption.

3. That the crystal lattice can be neglected. This assumption is clearly wrong for heterogeneous nucleation on surfaces which are not planar, e.g., wedges ${ }^{123}$. In wedges the nucleation of crystalline and fluid phases is qualitatively different. Also, where there is a lattice there are defects. Despite a large literature on the very large effects defects have on crystal growth, very 
little is known about the role defects play in nucleation. As we commonly study crystallisation under conditions where crystal growth is dominated by defects, it seems very likely that defects are playing a role in nucleation in some systems. It is just that we have not considered this. Simulation work on the role of defects in nucleation is urgently needed.

4. There is no other source of slow kinetics other than that due to the free energy barrier. Defects are a source of slow kinetics so if defects do form before the top of the nucleation barrier is reached there will be at least one source of slow dynamics in the nucleus. Also, Sanz et al. ${ }^{129,130}$ found that slow charge-ordering dynamics could change the polymorph that nucleated. In many systems crystallisation competes with slow (glassy) dynamics, for example, ACC appears to have very slow dynamics, and in many molecular systems crystallisation competes with the liquid becoming glassy. In these systems, the rate and location of nucleation may be dictated as much by where the dynamics are fastest (e.g., at a surface or near a heterogeneity which facilitates the molecular dynamics) as by where the nucleation barrier is lowest.

5. That the nucleation rate does not depend on the history of the sample. This assumption can be violated when there are glassy dynamics. It can also fail when there are surfaces present with nanoscale pits or pores, that can nucleate crystals and then retain crystals even above the melting point of the crystal in the bulk. This has been known for decades ${ }^{167}$. It is suspected that seed crystals can persist, possibly in pores, and then dramatically effect the nucleation behaviour ${ }^{7,10}$. More recently, there has been considerable work on hysteresis in porous media $^{183}$. Most of this is on vapour/liquid transitions but crystallisation in porous media has been considered, for example see the work of Radhakrishnan et al. ${ }^{184}$ and Christenson's review $^{185}$. This is all for porous media but if a bulk solution is in contact with a porous media then hysteresis in the porous media may cause history dependence in the crystallisation of the solution. The pores need not be a bulk porous media, it could be in the form of a nanoscale impurity in the solution ${ }^{112}$. History dependent nucleation is also clearly important in some applications of crystallisation, such as heat packs, where the heat is generated by exothermic crystallisation $^{186,187}$.

6. Nucleation occurs over a saddle point in the free energy, i.e., the critical nucleus is at a saddle point on the free-energy landscape. This means that although the free energy of the nucleus is a maximum with respect to one variable, its size, it is a minimum with respect to other variables. In the presence of slow dynamics of one coordinate but not others, this is not true ${ }^{110}$. This is also not true for crystallisation in the system of Sanz et al. ${ }^{129,130}$. If defects form they may also result in nuclei where this assumption is broken.

One point should be made about non-classical nucleation. This is that classical nucleation theory will be at heart of our attempts to model it. This should be clear from the repeated use of classical nucleation theory in this review. Even when one of the assumptions of the classical theory of nucleation fails, the others may still be valid so the classical theory still describes most of the physics and is still useful. The lesson is not that the classical theory should be discarded, but that it should be taken as the beginning not the end of our understanding of the nucleation of crystalline phases.

\section{Acknowledgements}

It is a pleasure to acknowledge G. Nicolis and D. Maes for the invitation to their inspiring workshop in Brussels in the spring of 2010. I can only apologise that I was not able to write an article for them on time. I would also like to thank F. Meldrum for the invitation to write this review. This review benefited greatly from a CECAM workshop in Lausanne in July 2010. I would like to acknowledge ideas and inspiration from the participants of this workshop, and the help of E. Bernard. I would also like to thank J. Doye for useful discussions on the role of defects. The section on laser-induced 
nucleation would not have been possible without discussions with D. Frenkel, D. Andrews and S. Smith. I am also grateful to A. Page for simulation results shown in section on defects, for simulation configurations, and for a BiBTeX file. For permission to reproduce their figures, I would like to thank J. Anwar, R. Bowles, H. Christenson, M. Muschol, S. K. Poornachary, J. D. Rodriguez-Blanco, C. P. Royall, R. Shaw, V. Thalladi, G. Tribello, B. Trout, M. Ward and J. Yu.

\section{References}

[1] R. P. Sear: J. Phys. Cond. Matt., 2007, 19, 033101.

[2] K. F. Kelton and A. L. Greer: Nucleation in Condensed Matter: Application in Materials and Biology. Pergamon, 2010.

[3] P. G. Debenedetti: Metastable Liquids. Princeton University Press, Princeton, 1996.

[4] S. K. Poornachary, P. S. Chow and R. B. H. Tan: Crys. Growth Des., 2008, 8, 179.

[5] S. K. Poornachary, P. S. Chow, R. B. H. Tan and R. J. Davey: Crys. Growth Des., 2007, 7, 254.

[6] J. Bauer, S. Spanton, R. Henry, J. Quick, W. Dziki, W. Porter and J. Morris: Pharm. Res., 2001, 18, 859 .

[7] S. L. Morisette, S. Soukasene, D. Levinson, M. J. Cima and O. Almarsson: Proc. Nat. Acad. Sci., 2003, 100, 2180.

[8] N. Variankaval, A. S. Cote and M. F. Doherty: AIChE Journal, 2008, 54.

[9] M. Prashad, P. Sutton, R. Wu, B. Hu, J. Vivelo and J. Carosi: Org. Process Res. Dev., 2010, 14, 878 .

[10] J. D. Dunitz and J. Bernstein: Acc. Chem. Res., 1995, 28, 193.

[11] A. S. Parmar, P. E. Gottschall and M. Muschol: Biophys. Chem., 2007, 129, 224.

[12] J. H. Seinfeld and S. N. Pandis: Atmospheric Chemistry and Physics. Wiley-Interscience, New York, 1998.

[13] H. R. Pruppacher and J. D. Klett: Microphysics of Clouds and Precipitation. Reidel Publishing, Dordrecht, 1978.

[14] B. J. Mason: The Physics of Clouds. Clarendon Press, Oxford, 1971.

[15] D. A. Hegg and M. B. Baker: Rep. Prog. Phys., 2009, 72, 056801.

[16] W. Cantrell and A. Heymsfield: Bull. Am. Meteor. Soc., 2005, 86, 795.

[17] B. J. Murray, D. A. Knopf and A. K. Bertram: Nature, 2005, 434, 202.

[18] B. J. Murray, S. L. Broadley, T. W. Wilson, S. J. Bull, R. H. Wills, H. K. Christenson and E. J. Murray: Phys. Chem. Chem. Phys., 2010, 12, 10380.

[19] A. J. Durant, R. A. Shaw, W. I. Rose, Y. Mi and G. G. J. Ernst: J. Geophys. Res., 2008, 113, D09206.

[20] N. H. Fletcher: J. Meteor., 1959, 16, 173.

[21] C. Gurganus, A. B. Kostinski and R. A. Shaw: J. Phys. Chem. Lett., 2011, 2, 1449. 
[22] A. J. Durant and R. A. Shaw: Geophys. Res. Lett., 2005, 109, L20814.

[23] R. A. Shaw, A. J. Durant and Y. Mi: J. Phys. Chem. B, 2005, 109, 9865.

[24] S. Suzuki, A. Nakajima, N. Yoshida, M. Sakai, A. Hashimoto, Y. Kameshima and K. Okada: Chem. Phys. Lett., 2007, 445, 37.

[25] S. Suzuki, A. Nakajima, N. Yoshida, M. Sakai, A. Hashimoto, Y. Kameshima and K. Okada: Langmuir, 2007, 23, 8674.

[26] A. Tabazadeh, Y. Djikaev and H. Reiss: Proc. Nat. Acad. Sci., 2002, 99, 15873.

[27] Y. Djikaev, A. Tabazadeh and H. Reiss: J. Chem. Phys., 2003, 118, 6572.

[28] M. P. Goertz, X. Y. Zhu and J. E. Houston: Langmuir, 2009, 25, 6905.

[29] Y. Li and G. A. Somorjai: J. Phys. Chem. C, 2007, 111, 9631.

[30] J. G. Dash, H. Fu and J. S. Wettlaufer: Rep. Prog. Phys., 1995, 58, 115.

[31] J. S. Rowlinson and B. Widom: Molecular Theory of Capillarity. Dover, Mineola, New York, 2002 .

[32] C. Vega, J. L. F. Abascal, M. M. Conde and J. L. Aragones: Faraday Disc., 2009, 141, 251.

[33] C. Vega, E. Sanz and J. L. F. Abascal: J. Chem. Phys., 2005, 122, 114507.

[34] V. Molinero and E. B. Moore: J. Phys. Chem. B, 2009, 113, 4008.

[35] A. V. Brukhno, J. Anwar, R. Davidchack and R. Handel: J. Phys, Cond, Matt., 2008, 20, 494243.

[36] D. Quigley and P. M. Rodger: J. Chem. Phys., 2008, 128, 154518.

[37] E. Sanz, C. Vega, J. L. F. Abascal and L. G. MacDowell: J. Chem. Phys., 2004, 121, 1165.

[38] E. Sanz, C. Vega, J. L. F. Abascal and L. G. MacDowell: Phys. Rev. Lett., 2004, 92, 255701.

[39] E. B. Moore and V. Molinero: J. Chem. Phys., 2010, 132, 244504.

[40] C. Valeriani, E. Sanz and D. Frenkel: J. Chem. Phys., 2005, 122, 194501.

[41] T. Zykova-Timan, C. Valeriani, E. Sanz, D. Frenkel and E. Tosatti: Phys. Rev. Lett., 2008, 100.

[42] H. Pan, X. Y. Liu, R. Tang and H. Y. Xu: Chem. Commun., 2010, 46, 7415.

[43] H. Cölfen and M. Antonietti: Mesocrystals and Nonclassical Crystallization. Wiley, Chichester, 2008 .

[44] F. C. Meldrum and H. Cölfen: Chem. Rev., 2008, 108, 4332.

[45] S. Mann: Biomineralization. Oxford University Press, Oxford, 2001.

[46] P. Raiteri, J. D. Gale, D. Quigley and P. M. Rodger: J. Phys. Chem. C, 2010, 114, 5997.

[47] P. Raiteri and J. D. Gale: J. Am. Chem. Soc., 2010, 132, 17623.

[48] J. D. Rodriguez-Blanco, S. Shaw and L. G. Benning: Nanoscale, 2011, 3, 265.

[49] F. M. Michel, J. MacDonald, J. Feng, B. L. Phillips, L. Ehm, C. Tarabrella, J. B. Parise and R. J. Reeder: Chem. Mat., 2008, 20, 4720. 
[50] A. L. Goodwin, F. M. Michel, B. L. Phillips, D. A. Keen, M. T. Dove and R. J. Reeder: Chem. Mater., 2010, 22, 3197.

[51] L. Addadi, S. Raz and S. Weiner: Adv. Mat., 2003, 15, 959.

[52] S. Weiner, J. Mahamid, Y. Politi, Y. Ma and L. Addadi: Frontiers of Materials Science in China, 2009, 3, 104.

[53] C. J. Stephens, S. F. Ladden, F. C. Meldrum and H. K. Christenson: Adv. Func. Mat., 2010, 20, 2108.

[54] C. J. Stephens, Y.-Y. Kim, S. D. Evans, F. C. Meldrum and H. K. Christenson: J Am. Chem. Soc., 2011, 133, 5210.

[55] C. L. Klix, K. Murata, H. Tanaka, S. R. Williams, A. Malins and C. P. Royall: arXiv, 2009, cond-mat/0905.3393.

[56] D. Gebauer, A. Völkel and H. Cölfen: Science, 2008, 322, 1819.

[57] F. C. Meldrum and R. P. Sear: Science, 2008, 322, 1802.

[58] E. M. Pouget, P. H. H. Bomans, J. A. C. M. Goos, P. M. Frederik, G. de With and N. A. J. M. Sommerdijk: Science, 2009, 323, 1455.

[59] F. Nudelman, E. Sonmezler, P. H. H. Bomans, G. de With and N. A. J. M. Sommerdijk: Nanoscale, 2010, 2, 2436.

[60] A. J. Bray: Adv. Phys., 1994, 43, 357.

[61] L. J. Lewis, P. Jensen and J.-L. Barrat: Phys. Rev. B, 1997, 56, 2248.

[62] P. Mirabel, H. Reiss and R. K. Bowles: J. Chem. Phys., 2000, 113, 8200.

[63] R. P. Sear: Europhys. Lett., 2008, 83, 66002.

[64] S. B. Hutchens and Z.-G. Wang: J. Chem. Phys., 2007, 127, 084912.

[65] J. F. Joanny and L. Leibler: J. Phys. France, 1990, 51, 545.

[66] J. A. van Meel, A. J. Page, R. P. Sear and D. Frenkel: J. Chem. Phys., 2008, 129, 204505.

[67] G. A. Tribello, F. Bruneval, C. Liew and M. Parrinello: J. Phys. Chem. B, 2009, 113, 11680.

[68] D. Quigley and P. M. Rodger: J. Chem. Phys., 2008, 128, 221101.

[69] D. Quigley, C. L. Freeman, J. H. Harding and P. M. Rodger: J. Chem. Phys., 2011, 134, 044703.

[70] E. B. Sirota and A. B. Herhold: Science, 1999, 283, 529.

[71] C. E. Hughes and K. D. M. Harris: Chem. Comm., 2010, 46, 4982.

[72] D. Kashchiev and K. Sato: 1998, 109, 8530.

[73] E. Morales-Sánchez, B. Laine, E. Prokhorov, G. Trapaga and J. Gonzlez-Hernndez: Materials Science and Engineering: A, 2004, 375-377, 763 . jce:title¿Eleventh International Conference on Rapidly Quenched and Metastable Materialsi/ce:titlę.

[74] B. Laine, G. Trapaga, E. Prokhorov, E. Morales-Snchez and J. Gonzlez-Hernndez: 2003, 83, 4969. 
[75] H.-J. Butt, K. Graf and M. Kappl: Physics and Chemistry of Interfaces. Wiley, Chichester, 2006.

[76] A. J. Page and R. P. Sear: Phys. Rev. E, 2009, 80, 031605.

[77] J. R. Cox, M. Dabros, J. A. Shaffer and V. R. Thalladi: Ang. Chem. Int. Ed., 2007, 46, 1988.

[78] L. Yu: Acc. Chem. Res., 2010, 43, 1257.

[79] S. Chen, H. Xi and L. Yu: J. Am. Chem. Soc., 2005, 127, 17439.

[80] P. R. ten Wolde and D. Frenkel: Phys. Chem. Chem. Phys., 1999, 1, 2191.

[81] W. Ostwald: Z. Phys. Chem., 1897, 289, 1897.

[82] L. Yu: J. Am. Chem. Soc., 2003, 125, 6380.

[83] L. Yu: Cryst. Eng. Comm., 2007, 9, 847.

[84] C. Cashell, D. Corcoran and B. K. Hodnett: Chem. Comm., 2003, 374.

[85] C. Cashell, D. Sutton, D. Corcoran and B. K. Hodnett: Crys. Growth Des., 2003, 3, 869.

[86] H. Mayama: Soft Matter, 2009, 5, 856.

[87] J. Tao, K. J. Jones and L. Yu: Crys. Growth Des., 2007, 7, 2410.

[88] C. Desgranges and J. Delhommelle: J. Am. Chem. Soc., 2006, 128, 15104.

[89] C. Desgranges and J. Delhommelle: J. Chem. Phys., 2007, 126, 054501.

[90] C. Desgranges and J. Delhommelle: J. Phys. Chem. B, 2007, 111, 12257. PMID: 17918891.

[91] A. R. Browning, M. F. Doherty and G. H. Fredrickson: Phys. Rev. E, 2008, 77, 041604.

[92] M. Muschol and F. Rosenberger: J. Chem. Phys., 1997, 107, 1953.

[93] O. Galkin and P. G. Vekilov: Proc. Nat. Acad. Sci., 2000, 97, 6277.

[94] W. Pan, A. B. Kolomeisky and P. G. Vekilov: J. Chem. Phys., 2005, 123, 014904.

[95] A. M. Streets and S. R. Quake: Phys. Rev. Lett., 2010, 104, 178102.

[96] P. E. Bonnett, K. J. Carpenter, S. Dawson and R. J. Davey: Chem. Comm., 2003, 698.

[97] W. H. Wang, C. Dong and C. H. Shek: Mat. Sci. Eng.: R: Rep., 2004, 44, 45.

[98] A. Gebert, J. Eckert and L. Schultz: Acta Metal., 1998, 46, 5475.

[99] T. H. Zhang and X. Y. Liu: J. Am. Chem. Soc., 2007, 129, 13520.

[100] J. R. Savage and A. D. Dinsmore: Phys. Rev. Lett., 2009, 102, 198302.

[101] A. C. Dumetz, A. M. Chockla, E. W. Kaler and A. M. Lenhoff: Biophys. J., 2008, 94, 570 .

[102] S. Janbon, R. J. Davey and G. Dent: J. Phys. Chem. C, 2008, 112, 15771.

[103] B. Chen, H. Kim, S. J. Keasler and R. B. Nellas: J. Phys. Chem. B, 2008, 112, 4067.

[104] N. Duff and B. Peters: J. Chem. Phys., 2009, 131, 184101.

[105] N. Duff and B. Peters: J. Chem. Phys., 2010, 132, 129901. 
[106] S. Whitelam: Phys. Rev. Lett., 2010, 105, 088102.

[107] R. Ohnesorge, H. Löwen and H. Wagner: Phys. Rev. E, 1994, 50, 4801.

[108] E. Mendez-Villuendas and R. K. Bowles: Phys. Rev. Lett., 2007, 98, 185503.

[109] E. Mendez-Villuendas and R. K. Bowles: Phys. Rev. Lett., 2007, 99, 159901.

[110] R. P. Sear: J. Chem. Phys., 2009, 131, 074702.

[111] S. Chattopadhyay, D. Erdemir, J. M. B. Evans, J. Ilavsky, H. Amenitsch, C. U. Segre and A. S. Myerson: Crys. Growth Des., 2005, 5, 523.

[112] P. Asanithi, E. Saridakis, L. Govada, I. Jurewicz, E. W. Brunner, R. Ponnusamy, J. A. S. Cleaver, A. B. Dalton, N. E. Chayen and R. P. Sear: ACS App. Mat. Inter., 2009, 1, 1203.

[113] P. Vekilov: Crys. Growth Des., 2004, 4, 671.

[114] O. Gliko, W. Pan, P. Katsonis, N. Neumaier, O. Galkin, S. Weinkauf and P. G. Vekilov: J. Phys. Chem. B, 2007, 111, 3106.

[115] P. G. Vekilov: Nanoscale, 2010, 2, 2346.

[116] K. H: Trans. Faraday. Soc., 1936, 32, 1152.

[117] B. P. Binks and J. H. Clint: Langmuir, 2002, 18, 1270.

[118] R. P. Sear: J. Phys. Cond. Matt., 2007, 19, 466106.

[119] Y. S. Djikaev and E. Ruckenstein: J. Phys. Chem. A, 2008, 112, 11677.

[120] L. Schimmele and S. Dietrich: Euro. Phys. J. E, 2009, 30, 427. 10.1140/epje/i2009-10542-3.

[121] A. J. Page: A Computational Study of Crystallisation in the Presence of Disordered Porous Media. PhD thesis, University of Surrey, 2010.

[122] J. D. Honeycutt and H. C. Andersen: J. Phys. Chem., 1987, 91, 4950.

[123] A. J. Page and R. P. Sear: J. Am. Chem. Soc., 2009, 131, 17550.

[124] Jmol: an open-source Java viewer for chemical structures in 3D: http://www.jmol.org/.

[125] I. V. Markov: Crystal Growth for Beginners. World Scientific, Singapore, 2004.

[126] F. C. Frank: Discuss. Faraday Soc., 1949, 5, 48.

[127] Y. Xiang, H. Jiang, W. Cai and X. Shao: J. Phys. Chem. A, 2004, 108, 3586.

[128] D. J. Wales, J. P. K. Doye, A. Dullweber, M. P. Hodges, F. Y. Naumkin, F. Calvo, J. Hernndez-Rojas and T. F. Middleton: The Cambridge Cluster Database. http://wwwwales.ch.cam.ac.uk/CCD.html.

[129] E. Sanz, C. Valeriani, D. Frenkel and M. Dijkstra: Phys. Rev. Lett., 2007, 99, 055501.

[130] E. Sanz, C. Valeriani, T. Vissers, A. Fortini, M. E. Leunissen, A. van Blaaderen, D. Frenkel and M. Dijkstra: J. Phys. Cond. Matt., 2007, 20, 494247.

[131] R. P. Sear: J. Chem. Phys., 2008, 128, 214513.

[132] B. Peters: J. Chem. Phys., 2009, 131, 244103.

[133] D. J. Wales: Energy Landscapes. Cambridge University Press, Cambridge, 2003. 
[134] J. P. K. Doye and F. Calvo: J. Chem. Phys., 2002, 116, 8307.

[135] E. G. Noya and P. K. Doye: J. Chem. Phys., 2006, 124, 104503.

[136] I. Saika-Voivod, L. Poon and R. K. Bowles: arXiv.

[137] P. Labastie and R. L. Whetten: Phys. Rev. Lett., 1990, 65, 1567.

[138] W. Polak: Cryst. Res. Technol., 2007, 42, 1207.

[139] J. Anwar, P. K. Boateng, R. Tamaki and S. Odedra: Ang. Chem. Int. Ed., 2009, 48, 1596.

[140] M. Hermes: Hard Spheres Out of Equilibrium (PhD thesis). (University of Utrecht), 2010.

[141] B. O’Malley and I. Snook: Phys. Rev. Lett., 2003, 90, 085702.

[142] A. J. Page and R. P. Sear: Phys. Rev. Lett., 2006, 97, 065701.

[143] P. G. de Gennes: Rev. Mod. Phys., 1985, 57, 827.

[144] D. Bonn and D. Ross: Rep. Prog. Phys., 2001, 64, 1085.

[145] R. P. Sear: J. Phys. Cond. Matt., 2002, 14, 3693.

[146] M. A. Castro, S. M. Clarke, A. Inaba and R. K. Thomas: J. Phys. Chem. B, 1997, 101, 8878.

[147] B. M. Ocko, X. Z. Wu, E. B. Sirota, S. K. Sinha, O. Gang and M. Deutsch: Phys. Rev. E, 1997, 55, 3164 .

[148] M. Dijkstra: Phys. Rev. Lett., 2004, 93, 108303.

[149] B. B. Laird and R. L. Davidchack: J. Phys. Chem., 2007, 111, 15952.

[150] S. Auer and D. Frenkel: Phys. Rev. Lett., 2003, 91, 015703.

[151] C. J. Stephens, Y. Mouhamad, F. C. Meldrum and H. K. Christenson: Crys. Growth Des., 2010, 10, 734 .

[152] D. Turnbull and B. Vonnegut: Ind. Eng. Chem., 1952, 44, 1292.

[153] J. A. van Meel, R. P. Sear and D. Frenkel: Phys. Rev. Lett., 2010, 105, 205501.

[154] C. A. Sholl and N. H. Fletcher: Acta Met., 1970, 18, 1083.

[155] E. H. Hauge: Phys. Rev. A, 1992, 46, 4994.

[156] A. O. Parry, A. J. Wood and C. Rascon: J. Phys. Cond. Matt., 2001, 13, 4591.

[157] A. N. Jackson, A. D. Bruce and G. J. Ackland: Phys. Rev. E, 2002, 62, 036710.

[158] A. Fortini and M. Dijkstra: J. Phys. Cond. Matt., 2006, 18, L371.

[159] E. C. Oğuz, R. Messina and H. Löwen: Europhys. Lett., 2009, 86, 28002.

[160] C. A. Mitchell, L. Yu and M. D. Ward: J. Am. Chem. Soc., 2001, 123, 10830.

[161] P. W. Carter and M. D. Ward: J. Am. Chem. Soc., 1993, 115, 11521.

[162] S. J. Bonafede and M. D. Ward: J. Am. Chem. Soc., 1995, 117, 7853.

[163] A. Cacciuto, S. Auer and D. Frenkel: Nature, 2004, 428, 404. 
[164] Y. Diao, M. E. Helgeson, A. S. Myerson, T. A. Hatton, P. S. Doyle and B. L. Trout: J. Am. Chem. Soc., 2011, 133, 3756.

[165] N. E. Chayen, E. Saridakis and R. P. Sear: Proc. Nat. Acad. Sci., 2006, 103, 597.

[166] M. Sugahara, Y. Asada, Y. Morikawa, Y. Kageyama and N. Kunishima: Acta Crys. D, 2008, 64, 686 .

[167] D. Turnbull: J. Chem. Phys., 1950, 18, 198.

[168] P. R. ten Wolde and D. Frenkel: Science, 1997, 277, 1975.

[169] Y. Diao, A. S. Myerson, T. A. Hatton and B. L. Trout: Langmuir, 2011, 27, 5324.

[170] P. Kabath, P. Stöckel, A. Lindinger and H. Baumgärtel: J. Mol. Liq., 2006, 125, 204.

[171] O. Galkin and P. G. Vekilov: J. Phys. Chem. B, 1999, 103, 10965.

[172] Š. Selimović, Y. Jia and S. Fraden: Crys. Growth Des., 2009, 9, 1806.

[173] B. A. Garetz, J. E. Aber, N. L. Goddard, R. G. Young and A. S. Myerson: Phys. Rev. Lett., 1996, 77, 3475 .

[174] J. Matic, X. Sun, B. A. Garetz and A. S. Myerson: Crys. Growth Des., 2005, 5, 1565.

[175] J. Zaccaro, J. Matic, A. S. Myerson and B. A. Garetz: Crys. Growth Des., 2001, 1, 5.

[176] X. Sun, B. A. Garetz and A. S. Myerson: Crys. Growth Des., 2006, 6, 684.

[177] A. J. Alexander and P. J. Camp: Crys. Growth Des., 2009, 9, 958.

[178] B. C. Knott, M. F. Doherty and B. Peters: J. Chem. Phys., 2011, 134, 154501.

[179] J. Huang, T. C. Stringfellow and L. Yu: J. Am. Chem. Soc., 2008, 130, 13973.

[180] F. Hodzhaoglu, F. Kurniawan, V. Mirsky and C. Nanev: Crys. Res. Tech., 2008, 43, 588.

[181] H. Y. Yoshikawa, Y. Hosokawa and H. Masuhara: Jap. J. App. Phys., 2006, 45, L23.

[182] R. Murai, H. Y. Yoshikawa, Y. Takahashi, M. Maruyama, S. Sugiyama, G. Sazaki, H. Adachi, K. Takano, H. Matsumura, S. Murakami, T. Inoue and Y. Mori: App. Phys. Lett., 2010, 96, 043702 .

[183] L. D. Gelb, K. E. Gubbins, R. Radhakrishnan and M. Sliwinska-Bartkowiak: Rep. Prog. Phys., 1999, 62, 1573.

[184] R. Radhakrishnan, K. E. Gubbins and M. Sliwinska-Bartkowiak: J. Chem. Phys., 2002, 116, 3 .

[185] H. K. Christenson: J. Phys. Cond. Matt., 2001, 13, R95.

[186] M. A. Rogerson and S. S. S. Cardoso: AIChE J., 2003, 49, 505.

[187] M. A. Rogerson and S. S. S. Cardoso: AIChE J., 2003, 49, 516. 